\title{
Marangoni convection in binary mixtures with Soret effect
}

\author{
By A. BERGEON ${ }^{1}$, D. HENRY $Y^{2}$ H. BENHADID \\ AND L. S. TUCKERMA N \\ ${ }^{1}$ IMFT, UMR 5502 CNRS, Laboratoire de Mécanique des Fluides, Université Paul Sabatier, \\ 118 route de Narbonne, 31062 Toulouse Cedex, France. \\ ${ }^{2}$ Laboratoire de Mécanique des Fluides et d'Acoustique, UMR 5509, \\ Ecole Centrale de Lyon / Université Claude-Bernard Lyon 1, ECL, BP 163, \\ 69131 Ecully Cedex, France \\ ${ }^{3}$ Laboratoire d'Informatique pour la Mécanique et les Sciences de l'Ingénieur, BP 133, \\ 91403 Orsay Cedex, France
}

(Received 1 April 1997 and in revised form 22 June 1998)

\begin{abstract}
Marangoni convection in a differentially heated binary mixture is studied numerically by continuation. The fluid is subject to the Soret effect and is contained in a twodimensional small-aspect-ratio rectangular cavity with one undeformable free surface. Either or both of the temperature and concentration gradients may be destabilizing; all three possibilities are considered. A spectral-element time-stepping code is adapted to calculate bifurcation points and solution branches via Newton's method. Linear thresholds are compared to those obtained for a pure fluid. It is found that for large enough Soret coefficient, convection is initiated predominantly by solutal effects and leads to a single large roll. Computed bifurcation diagrams show a marked transition from a weakly convective Soret regime to a strongly convective Marangoni regime when the threshold for pure fluid thermal convection is passed. The presence of many secondary bifurcations means that the mode of convection at the onset of instability is often observed only over a small range of Marangoni number. In particular, two-roll states with up-flow at the centre succeed one-roll states via a well-defined sequence of bifurcations. When convection is oscillatory at onset, the limit cycle is quickly destroyed by a global (infinite-period) bifurcation leading to subcritical steady convection.
\end{abstract}

\section{Introduction}

The ability of temperature-induced surface tension gradients to drive a flow was established by Block (1956) and by Pearson (1958). Correcting an error that had lasted half a century, these authors established that the hexagonal convection cells observed by Bénard (1901) and explained by Rayleigh (1916) as being driven by thermal buoyancy, were in fact due instead to temperature-dependent surface tension. Convection driven by surface tension gradients is now called Marangoni convection (after an earlier observation) or Marangoni-Bénard convection, in contrast to buoyancy-driven Rayleigh-Bénard convection. Marangoni convection arises in many physical contexts involving multiphase flow. It has a significant influence on convective mass transport during the solidification of a liquid metal in floating zone configuration, particularly in a microgravity environment where buoyancy-driven convection is almost suppressed. 
Since the quality of the crystal depends on the concentration distribution in the liquid phase, it is important to understand and to control Marangoni convection.

In a typical configuration, a temperature gradient is imposed between two parallel bounding surfaces, one rigid and one free, of a fluid layer. A temperature perturbation along the free surface will cause the fluid to move along the surface away from regions of lower surface tension, and the perturbation will be damped or amplified according to the temperature of the fluid brought from the interior to conserve mass. Since the driving mechanism must overcome viscosity and thermal diffusion, the imposed temperature gradient, characterized by the dimensionless Marangoni number $M a$, must exceed a critical value for convection to occur.

The classical experiments in Marangoni convection are those of Koschmieder. In a series of papers spanning many years and geometries, Koschmieder and coworkers (Koschmieder 1967; Koschmieder \& Prahl 1990; Koschmieder \& Switzer 1992) described transitions in silicone oil to rolls, hexagons, or other planforms, depending on the shape (circular or square) and the thickness of the layer. Quantitative disagreements between experiment and theory have indicated that gravity or free surface deformation might play a significant role. Nield (1964), Davis (1969), Davis \& Homsy (1980), Kraska \& Sani (1979), Castillo \& Velarde (1978, 1982), Cloot \& Lebon (1984) and Oron \& Rosenau (1989) have addressed the effects of gravity by studying the combined effects of surface tension and of buoyancy - Rayleigh-Bénard-Marangoni convection - using energy or variational stability analyses and weakly nonlinear analyses. Scriven \& Sternling (1964), Smith (1966) and Goussis \& Kelly (1990) have refined Pearson's model by incorporating a more realistic interface.

Most of the above-mentioned theoretical work followed Pearson in assuming analytically tractable, horizontally infinite layers. The containers of finite size which are the subject of the present study must be treated numerically. We can distinguish further between free-slip sidewalls, whose main effect is to discretize the possible wavelengths, and more realistic rigid walls. A linear and weakly nonlinear study of Marangoni convection in cylindrical and rectangular containers with free-slip sidewalls was carried out by Rosenblat, Davis \& Homsy (1982). The linear problem of a rectangular container with rigid sidewalls was studied by Van de Vooren \& Dijkstra (1989), and extended to three dimensions by Dijkstra (1995).

The investigations which are closest in spirit to ours are those of Winters, Plesser \& Cliffe (1988) and of Dijkstra (1992). These studies produced bifurcation diagrams by numerical continuation of fully nonlinear Rayleigh-Bénard-Marangoni convection in two-dimensional rectangular containers. Both emphasized the role of lateral boundary conditions and symmetry. When the sidewalls are rigid, pitchfork (symmetry-breaking) and transcritical (symmetry-preserving) bifurcations occur in alternation as either aspect ratio or Marangoni number is increased, and the spatial structure of each eigenvector changes continuously. When the sidewalls are free-slip, all bifurcations are pitchforks and each eigenvector retains its spatial structure. Dijkstra calculated a number of secondary bifurcations and oscillatory states, as well as codimension-two bifurcations where two bifurcations coincide. Finally, Dauby \& Lebon (1997) and Thess \& Orszag (1995) have carried out numerical bifurcation studies for representative three-dimensional containers whose horizontal cross-section is rectangular or periodic, respectively.

The major focus of our study is the effect on Marangoni convection of the addition of a second fluid. Convection in binary fluids is considerably more complicated than that in pure fluids. Both temperature and concentration gradients contribute to the initiation of convection and each may be stabilizing or destabilizing. Even when a 
concentration gradient is not externally imposed (the thermosolutal problem) it can be created by the applied thermal gradient via the Soret effect. Thermosolutal and Soretinduced Rayleigh-Bénard convection have been shown to be formally equivalent by Knobloch (1980) when identical boundary conditions are imposed on temperature and concentration.

Rayleigh-Bénard convection in binary mixtures has been the subject of intense investigation over the last few decades. Early theoretical studies, e.g. Nield (1967), Veronis (1968), Hurle \& Jakeman (1971), Legros, Platten \& Poty (1972), Lhost \& Platten (1989) and Schechter, Prigogine \& Hamm (1972) calculated critical Rayleigh numbers $R a$, wavenumbers, and frequencies as a function of a Soret coefficient $S$ (or some equivalent parameter). They predicted oscillations at onset and possibly subcritical steady convection when a stabilizing concentration gradient opposes a destabilizing temperature gradient, i.e. for $R a$ positive and $S$ sufficiently negative. Steady convection is predicted when the concentration gradient is destabilizing, i.e. when $R a$ and $S$ are of the same sign, with a critical wavenumber of zero for $|S|$ sufficiently large.

These interesting features led Rayleigh-Bénard convection with Soret effect to be adopted as a paradigm for new developments in the theory of dynamical systems and pattern formation in the 1980s. Brand, Hohenberg \& Steinberg (1984) and Knobloch (1986) considered the simultaneous onset of oscillatory and stationary instabilities as a codimension-two point, and Cross (1988) studied the effect of confinement of travelling waves. During the same period, experiments were performed (e.g. Walden, Kolodner \& Passner 1985; Rehberg \& Ahlers 1985; Moses, Fineberg \& Steinberg 1987; Heinrichs, Ahlers \& Cannell 1987; Bensimon et al. 1990; Zimmermann, Muller \& Davis 1992) which displayed the phenomena predicted and analyzed by theorists, such as vanishing frequencies, multistability, defects, and localized or chaotic travelling waves. One obstacle faced in comparing theory and experiment is the notorious difficulty of determining the Soret coefficient; this is addressed in theoretical studies by Henry \& Roux (1988) and Knobloch \& Moore (1988). The current state of knowledge concerning Rayleigh-Bénard convection with Soret effect is summarized in a comprehensive study by Barten et al. (1995).

This vast literature contrasts with the paucity of studies on Marangoni convection in binary mixtures. Stability analyses in horizontally infinite layers of thermosolutal Marangoni convection have been carried out by McTaggart (1983) and Ho \& Chang (1988) and of Marangoni convection with Soret effect by Castillo \& Velarde (1978, 1982) and by Bergeon, Henry \& BenHadid (1995). These analyses were extended to include other effects, such as weak gravity (Chen \& Chen 1994), a transient concentration profile (Van Vaerenbergh et al. 1991), and surface deflection (Joo 1995). These studies indicate that, in spite of the very different physical mechanisms involved, surface-tension-driven Marangoni convection in a binary mixture with Soret effect shares many of the features of its buoyancy-driven analogue. In particular, as will be reviewed in $\S 4$, the critical wavenumber for steady convection is zero when the solutal contribution to the surface tension is sufficiently destabilizing and the first instability is a Hopf bifurcation to traveling or standing waves when a sufficiently stabilizing solutal contribution opposes a destabilizing thermal contribution.

In preliminary investigations of bounded cavities, Bergeon, Henry \& BenHadid (1994a) and Bergeon et al. (1994b) observed the finite-size analogues of phenomena predicted in horizontally infinite layers. In particular, Bergeon et al. (1994b) observed a roll which is as large as the container permits, and Bergeon et al. (1994a) computed a time-periodic flow in which infinitesimal rolls appeared, grew, and displaced other rolls. However, to our knowledge, there exists no comprehensive survey of nonlinear 


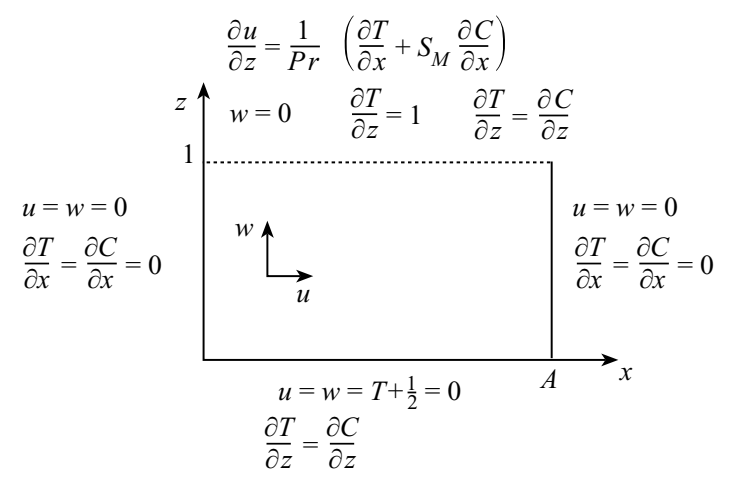

Figure 1. Cavity configuration.

Marangoni convection in a binary fluid with Soret effect in bounded cavities. The purpose of this paper is to bridge this gap by computing thresholds and bifurcation diagrams for a wide variety of Soret coefficients. We will consider a fluid with the moderate Prandtl number and high Schmidt number typical of molten salts or liquid metals. The precise values $(P r=0.6, S c=60)$ will be those of the AgI-KI mixture described in Henry \& Roux (1988).

We have simplified the geometry in two important ways. First, the cavity is twodimensional. This is realistic for containers with a small width compared to the length, where two-dimensional patterns are indeed observed (Davis 1987). In addition, the study of two-dimensional patterns provides a stepping stone to a better understanding of the three-dimensional case. Secondly, the free surface is considered undeformed by the flow. This assumption is justified when the surface tension and gravity may resist the flow-induced free surface deformation, but may fail far from the threshold when the flow intensity becomes large.

We bring to bear on this problem a full range of numerical methods, all based on the same spectral element temporal-integration code. This code is adapted to carry out Newton's method, first, to calculate bifurcations from the conductive state, and second, to calculate stable and unstable branches of steady states. Linear stability analysis and time-integration complete the picture by locating Hopf bifurcations and computing oscillatory states.

The paper is organized as follows. In $\S 2$, the equations for Marangoni convection in a binary mixture with Soret effect are presented, and the relevant dimensionless parameters defined. In $\S 3$, we describe our numerical methods, in particular the implementation of Newton's method which is our primary tool. In $\S 4$, we present convection thresholds calculated for a variety of aspect ratios and Soret coefficients, and compare them to thresholds obtained in horizontally infinite layers and in the limiting cases of a pure thermal and a pure solutal problem. In $\S 5$, we present bifurcation diagrams for representative Soret coefficients and compare them to the bifurcation diagrams for the pure thermal case. In our conclusion, $\S 6$, we summarize the general principles that have emerged from our study.

\section{Governing equations}

We consider a binary fluid layer contained in a rectangular cavity of length $L$ and height $H$ (see figure 1). A constant and uniform normal heat flux $q$ is applied to the 
upper free surface. The lower rigid boundary is maintained at a constant temperature $T_{0}$.

The inclusion of the Soret effect means that the mass flux is the sum of temperature and concentration gradients (De Groot \& Mazur 1969). (We neglect the Dufour effect, by which the concentration gradient would contribute to the heat flux.) The fluxes are then:

$$
\begin{gathered}
\boldsymbol{J}_{C}=-\rho D \nabla C-\rho D_{S} \nabla T, \\
\boldsymbol{J}_{T}=-D_{T} \nabla T,
\end{gathered}
$$

where $\rho$ is the density, and $D, D_{S}, D_{T}$ are the mass diffusion coefficient, the Soret diffusion coefficient and thermal conductivity, respectively. The applied heat flux generates a temperature difference $\Delta T=-q H / D_{T}$ and, via the Soret effect, a concentration difference $\Delta C=-D_{S} \Delta T / D$ over the height of the layer. At mid-layer, the temperature is $T_{m}=T_{0}+\Delta T / 2$ and the concentration is the initial mass fraction $C_{m}$. The Soret diffusion coefficient $D_{S}$ is in principle proportional to $C(1-C)$, but we approximate $C(1-C) \approx C_{m}\left(1-C_{m}\right)$, assuming that the Soret effect is weak.

The free surface is flat and subject to surface tension. The surface tension $\sigma$ is assumed to vary linearly with the temperature and the mass fraction:

$$
\sigma(T, C)=\sigma_{m}\left[1+\gamma_{T}\left(T-T_{m}\right)+\gamma_{C}\left(C-C_{m}\right)\right]
$$

with $\gamma_{T}$ and $\gamma_{C}$ constant. The surface tension is characterized by two non-dimensional quantities: the usual Marangoni number $M a$, and the Marangoni Soret parameter $S_{M}$, which we define by analogy to the separation constant for buoyancy-driven convection and which represents the ratio of the solutal contribution to the thermal contribution to the surface tension. These are given by

$$
M a=\frac{\Delta T H \sigma_{m} \gamma_{T}}{\rho v \kappa} \quad \text { and } \quad S_{M}=\frac{-D_{S} \gamma_{C}}{D \gamma_{T}},
$$

where $v$ is the kinematic viscosity and $\kappa$ is the thermal diffusion coefficient.

The velocity field is $\boldsymbol{U}=(u, 0, w)$. Distance, time, and velocity are nondimensionalized by $H, H^{2} / v$, and $M a v / H$, respectively. (The reason for this non-standard choice of non-dimensionalization is the convenience for numerical continuation of having $M a$ appear in the equations rather than in the boundary conditions; see §3.2.) The dimensionless temperature and concentration are taken to be $\left(T-T_{m}\right) / \Delta T$ and $\left(C-C_{m}\right) / \Delta C$, hereafter denoted by $T$ and $C$. The equations by which $\boldsymbol{U}, T, C$ and the pressure $p$ evolve result from the conservation laws for an incompressible fluid:

$$
\begin{gathered}
\frac{\partial \boldsymbol{U}}{\partial t}=-M a(\boldsymbol{U} \cdot \nabla) \boldsymbol{U}+\nabla^{2} \boldsymbol{U}-\nabla p, \\
\frac{\partial T}{\partial t}=-M a(\boldsymbol{U} \cdot \nabla) T+\frac{1}{P r} \nabla^{2} T, \\
\frac{\partial C}{\partial t}=-M a(\boldsymbol{U} \cdot \nabla) C+\frac{1}{S c}\left(\nabla^{2} C-\nabla^{2} T\right), \\
0=\nabla \cdot \boldsymbol{U},
\end{gathered}
$$

where $\operatorname{Pr}=v / \kappa$ and $S c=v / D$ are the Prandtl and the Schmidt number, respectively. Throughout this paper, we fix $\operatorname{Pr}=0.6$ and $S c=60$.

The aspect ratio of the cavity is $A=L / H$. We impose no-slip boundary conditions along the rigid bottom and lateral walls. The lateral walls are insulating, while a 
constant temperature is maintained on the bottom. Across all the rigid boundaries, the normal mass flux is zero. Along the lateral walls $x=0, A$, we thus obtain

$$
u=w=\frac{\partial(C-T)}{\partial x}=\frac{\partial T}{\partial x}=0
$$

and along the rigid bottom $z=0$,

$$
u=w=\frac{\partial(C-T)}{\partial z}=0, \quad T=-0.5 .
$$

Along the free surface, the normal velocity $w$ is zero and the stress equilibrium gives the boundary condition for the horizontal velocity $u$. The normal heat flux is constant and the normal mass flux is zero. The resulting boundary conditions at $z=1$ are

$$
\frac{\partial u}{\partial z}-\frac{1}{P r}\left(\frac{\partial T}{\partial x}+S_{M} \frac{\partial C}{\partial x}\right)=w=\frac{\partial(C-T)}{\partial z}=0 \quad \frac{\partial T}{\partial z}=1 .
$$

\section{Numerical methods}

We used two numerical methods to investigate this problem: time integration and continuation. The time integration scheme uses a spectral element method and is described in the following first sub-section. The continuation method, described in the next three sub-sections, is based on the time integration scheme. We use it to follow solution branches as a function of Marangoni number, as well as to directly calculate critical Marangoni numbers as a function of the Marangoni Soret parameter $S_{M}$ and the aspect ratio $A$.

\subsection{Time stepping}

The fields are discretized via the spectral element method (Patera 1984), i.e. as values on the Gauss-Lobatto-Chebyshev points and also as series of Chebyshev polynomials.

The linear terms are integrated implicitly and the nonlinear terms explicitly:

$$
\begin{aligned}
& \frac{\boldsymbol{U}^{(n+1)}-\boldsymbol{U}^{(n)}}{\Delta t}=-M a\left(\boldsymbol{U}^{(n)} \cdot \nabla\right) \boldsymbol{U}^{(n)}-\nabla p^{(n+1)}+\nabla^{2} \boldsymbol{U}^{(n+1)}, \\
& \frac{T^{(n+1)}-T^{(n)}}{\Delta t}=-M a\left(\boldsymbol{U}^{(n)} \cdot \nabla\right) T^{(n)}+\frac{1}{P r} \nabla^{2} T^{(n+1)}, \\
& \frac{C^{(n+1)}-C^{(n)}}{\Delta t}=-M a\left(\boldsymbol{U}^{(n)} \cdot \nabla\right) C^{(n)}+\frac{1}{S c}\left(\nabla^{2} C^{(n+1)}-\nabla^{2} T^{(n+1)}\right) .
\end{aligned}
$$

After the nonlinear terms have been computed, a Poisson problem is formulated for the pressure, using the boundary conditions given by Karniadakis, Israeli \& Orszag (1991). This Poisson problem, as well as the Helmholtz problems which constitute the final implicit step of the scheme are solved using a variational formulation.

The first-order time-stepping scheme (3.1) will be adapted for steady-state solving as described in the next sub-section. When time-stepping itself is the goal, as in the computation of a limit cycle, the second- or third-order accurate schemes described in Karniadakis et al. (1991) are used.

\subsection{Steady-state solving}

The time-stepping code is modified as described in Tuckerman (1989) and Mamun \& Tuckerman (1995) to compute steady states by Newton's method. We begin by 
re-writing (3.1) in the abbreviated form

$$
\frac{\boldsymbol{X}^{(n+1)}-\boldsymbol{X}^{(n)}}{\Delta t}=\operatorname{Ma} \mathbf{N} \boldsymbol{X}^{(n)}+\boldsymbol{L} \boldsymbol{X}^{(n+1)} .
$$

Here, $\boldsymbol{X}$ represents all of the spatially discretized fields $(\boldsymbol{U}(u, w), T, C), \boldsymbol{N}$ and $\boldsymbol{L}$ represent the spatially discretized nonlinear and linear operators, and $M a$ is the Marangoni number. (The pressure does not appear in $\boldsymbol{X}$ because it does not obey a time evolution equation but instead can in principle be computed from the variables in $\boldsymbol{X}$ by imposing incompressibility of the velocity.) The time-stepping scheme (3.2) can, after some algebra (see Tuckerman 1989; Mamun \& Tuckerman 1995), be rewritten as

$$
\frac{\boldsymbol{X}^{(n+1)}-\boldsymbol{X}^{(n)}}{\Delta t}=(\boldsymbol{I}-\Delta t \boldsymbol{L})^{-1}(M a \boldsymbol{N}+\boldsymbol{L}) \boldsymbol{X}^{(n)} .
$$

Now we consider the steady-state problem

$$
0=\operatorname{MaN} \boldsymbol{X}+\boldsymbol{L} \boldsymbol{X} .
$$

To use Newton's method on (3.4), at each step we must solve

$$
\begin{aligned}
\left(\operatorname{MaN}_{\boldsymbol{X}}+\boldsymbol{L}\right) \boldsymbol{\delta} \boldsymbol{X} & =-(\operatorname{MaN}+\boldsymbol{L}) \boldsymbol{X}, \\
\boldsymbol{X} & \leftarrow \boldsymbol{X}+\delta \boldsymbol{X},
\end{aligned}
$$

where $\boldsymbol{N}_{\boldsymbol{X}}$ is the Jacobian of $\boldsymbol{N}$ evaluated at $\boldsymbol{X}$. Instead of solving (3.5), we solve

$$
(\boldsymbol{I}-\Delta t \boldsymbol{L})^{-1}\left(M a \boldsymbol{N}_{\boldsymbol{X}}+\boldsymbol{L}\right) \boldsymbol{\delta} \boldsymbol{X}=-(\boldsymbol{I}-\Delta t \boldsymbol{L})^{-1}(M a \boldsymbol{N}+\boldsymbol{L}) \boldsymbol{X} .
$$

For large $\Delta t$, the operator $\boldsymbol{P} \equiv(\boldsymbol{I}-\Delta t \boldsymbol{L})^{-1}$ serves as a preconditioner (i.e. approximate inverse) for $M a \boldsymbol{N}+\boldsymbol{L}$, greatly accelerating iterative inversion. In practice, we use $\Delta t=10^{5}$.

If we solve the linear system (3.6) by an iterative conjugate gradient method, we need only provide the right-hand side and the action of the matrix-vector product constituting the left-hand side. Referring to (3.3), we see that the right-hand side of (3.6) can be obtained by carrying out a time step, and the matrix-vector product by carrying out a linearized time step. We emphasize that the Jacobian matrix is never constructed or stored. We solve equation (3.6) with the biconjugate gradient squared (BCGS) algorithm from the NSPCG (Oppe, Joubert \& Kincaid 1989) software library.

\subsection{Continuation}

In the above, the unknowns $\boldsymbol{X}=(\boldsymbol{U}, T, C)$ are determined as a function of a fixed $M a$. At a saddle-node or pitchfork bifurcation, $\boldsymbol{X}$ ceases to be a function of $M a$; in order to follow a solution branch, we must be prepared to calculate a more general curve in $(\boldsymbol{X}, M a)$. When any component $\boldsymbol{X}_{l}$ varies faster than some threshold, we treat this as an imminent bifurcation by fixing $\boldsymbol{X}_{l}$ and allowing $M a$ to vary. One Newton step for solving (3.4) becomes

$$
\begin{gathered}
{\left[\begin{array}{cc}
M a \mathbf{N}_{\boldsymbol{X}}+\boldsymbol{L} & \boldsymbol{N} \boldsymbol{X} \\
\mathrm{e}_{l}^{T} & 0
\end{array}\right]\left[\begin{array}{c}
\delta \boldsymbol{X} \\
\delta M a
\end{array}\right]=-\left[\begin{array}{c}
(\operatorname{Ma} \boldsymbol{N}+\boldsymbol{L}) \boldsymbol{X} \\
0
\end{array}\right],} \\
\boldsymbol{X} \leftarrow \boldsymbol{X}+\delta \boldsymbol{X}, \\
M a \leftarrow M a+\delta M a .
\end{gathered}
$$


The last equation of the linear system (3.7), containing the transpose of the $l$ th unit vector, merely states that $\delta \boldsymbol{X}_{l}=0$ so that $\boldsymbol{X}_{l}$ remains fixed. Because $\delta \boldsymbol{X}_{l}=0$ is imposed, the unknowns are effectively a truncated $\delta \boldsymbol{X}$ containing all but its $l$ th component, and $\delta M a$. Thus the number of unknowns remains the dimension of $\boldsymbol{X}$, which allows virtually all of the structure of the time-stepping code to be preserved.

System (3.7) can be preconditioned in the same way as (3.5). We obtain

$$
\left[\begin{array}{cc}
\boldsymbol{P}\left(M a \mathbf{N}_{\boldsymbol{X}}+\boldsymbol{L}\right) & \boldsymbol{P} \boldsymbol{N} \boldsymbol{X} \\
\mathrm{e}_{l}^{T} & 0
\end{array}\right]\left[\begin{array}{c}
\delta \boldsymbol{X} \\
\delta M a
\end{array}\right]=-\left[\begin{array}{c}
\boldsymbol{P}(M a \mathbf{N}+\boldsymbol{L}) \boldsymbol{X} \\
0
\end{array}\right] .
$$

This preconditioned system can now be solved rapidly by conjugate gradient iteration. As before, both the right-hand side and the matrix-vector product in (3.8) are easily obtained by minor modification of the time integration scheme. (See Mamun and Tuckerman 1995 for more details.) The predictor - i.e. the initial guess for the Newton iteration - is evaluated by quadratic or linear extrapolation along the solution branch.

\subsection{Direct calculation of bifurcation points}

Once we have identified a bifurcation point with critical Marangoni number $M a_{c}$, we will be interested in tracing its evolution as we vary other parameters, here the Marangoni Soret parameter $S_{M}$ and the aspect ratio $A$. In fact, primary bifurcation points can be directly calculated by a variant of the continuation method we have just described.

At a steady bifurcation point, $\boldsymbol{X}$ is a solution to (3.4) and the Jacobian is singular, with a null vector $\boldsymbol{h}$ whose $l$ th component will be normalized to 1 :

$$
\begin{aligned}
(M a \boldsymbol{N}+\boldsymbol{L}) \boldsymbol{X} & =0, \\
\left(\mathrm{MaN}_{\boldsymbol{X}}+\boldsymbol{L}\right) \boldsymbol{h} & =0, \\
\boldsymbol{h}_{l}-1 & =0 .
\end{aligned}
$$

For a primary bifurcation point, $\boldsymbol{X}$ is the known conductive solution, and so (3.9a) need not be solved. One Newton step for solving $(3.9 b, c)$ is:

$$
\begin{aligned}
{\left[\begin{array}{cc}
M a \mathbf{N}_{\boldsymbol{x}}+\boldsymbol{L} & \boldsymbol{N}_{\boldsymbol{x}} \boldsymbol{h} \\
\mathrm{e}_{l}^{T} & 0
\end{array}\right]\left[\begin{array}{c}
\delta \boldsymbol{h} \\
\delta M a
\end{array}\right]=-\left[\begin{array}{c}
\left(M_{a} \boldsymbol{N}_{\boldsymbol{x}}+\boldsymbol{L}\right) \boldsymbol{h} \\
0
\end{array}\right], } \\
\boldsymbol{h} \leftarrow \boldsymbol{h}+\delta \boldsymbol{h}, \\
M a \leftarrow M a+\delta M a,
\end{aligned}
$$

which is identical to (3.7) except for the replacement of $\boldsymbol{N} \boldsymbol{X}$ by $\boldsymbol{N}_{\boldsymbol{X}} \boldsymbol{h}$. The system (3.10) is preconditioned and solved by adapting the time integration scheme for conjugate gradient iteration, exactly as described for steady-state solving and for continuation.

\subsection{Leading eigenvalues}

Finally, in order to determine if a solution is stable or unstable, we will occasionally calculate leading eigenvalues - those with largest real part and thus responsible for initiating instability - and their corresponding eigenvectors. To do so, we use the adaptation of Arnoldi's method described in Mamun \& Tuckerman (1995). By 


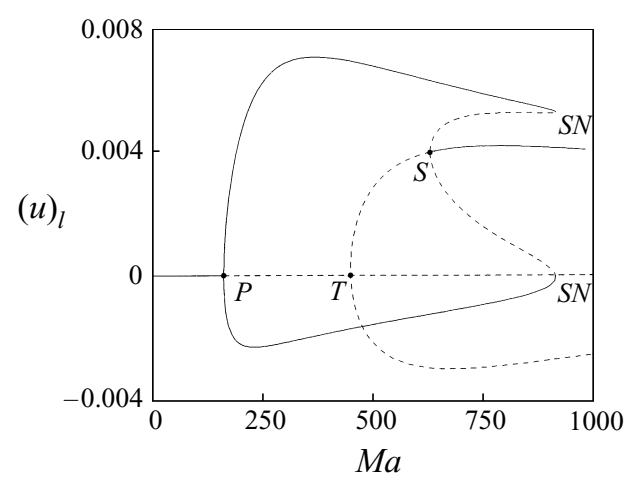

FiguRE 2. Bifurcation diagram for Marangoni convection in a pure fluid with $\operatorname{Pr}=8$ and $A=1$. Critical Marangoni numbers for primary pitchfork and transcritical bifurcations are $M a_{P}=161.6$ and $M a_{T}=450.4$; those for secondary pitchfork and saddle-node bifurcations are $M a_{S}=642.0$ and $M a_{S N}=933$.

time-stepping the linearized equations, we are able to construct a small matrix which represents the action of the Jacobian $\left(\boldsymbol{L}+\boldsymbol{M a \mathbf { N } _ { \boldsymbol { X } }}\right)$ on the subspace of leading eigenvectors. Diagonalization of this matrix yields the leading eigenvalues and eigenvectors. We have computed up to two real or complex leading eigenvalues to $1 \%$ accuracy.

\subsection{Accuracy and resolution}

To check the accuracy of the code we have computed the bifurcation diagram for a pure fluid with $A=1$ and $P r=8$, and compared it with that computed by Dijkstra (1992). Figure 2 shows the velocity $(u)_{l}$ at a representative point as a function of Marangoni number Ma. Using a resolution $11 \times 11$, we observe a pitchfork bifurcation $P$, a transcritical bifurcation $T$ (hysteresis not visible), saddle-node bifurcations $S N$, and a secondary pitchfork bifurcation $S$. We will present a number of bifurcation diagrams of this type in $\S 5$, and so we postpone a detailed description of the notation and phenomena on figure 2 . Here we merely note that the critical Marangoni numbers for all of these bifurcations differ by less than 3\% from those presented in Dijkstra (1992).

We have used spatial resolutions ranging from $11 \times 11$ up to $29 \times 15$ where necessary. We have validated the spectral element code by comparing its results with those calculated by an entirely independent time-stepping code (BenHadid \& Roux 1992) whose spatial discretization uses Hermitian finite differences on a $101 \times 21$ grid. For time stepping, we used $\Delta t=10^{-2}$.

\section{Linear stability analysis}

In this section, we present linear stability results for a number of cases. We will first summarize analytic results obtained previously (Bergeon et al. 1995) for an infinite horizontal layer. This is followed by the detailed numerical study of bounded twodimensional cavities, first for a pure fluid and then for binary mixtures. Our goal is to follow the evolution of convection thresholds from the pure thermal problem $\left(S_{M}=0\right)$ to a solutal-dominated regime $\left(S_{M}\right.$ larger).

The equations which will be considered throughout this section are obtained by linearizing equations (2.4)-(2.5) about the conductive solution $\bar{T}=\bar{C}=-0.5+z$. 
The resulting eigenvalue problem is

$$
\begin{aligned}
& \lambda \boldsymbol{U}=\nabla^{2} \boldsymbol{U}-\nabla p, \\
& \lambda T=-M a w+\frac{1}{P r} \nabla^{2} T, \\
& \lambda C=-M a w+\frac{1}{S c} \nabla^{2}(C-T), \\
& 0=\nabla \cdot \boldsymbol{U},
\end{aligned}
$$

with boundary conditions

$$
\begin{array}{ll}
u=w=T=\frac{\partial(C-T)}{\partial z}=0 & \text { at } z=0, \\
w=\frac{\partial T}{\partial z}=\frac{\partial(C-T)}{\partial z}=0 & \text { at } z=1, \\
\frac{\partial u}{\partial z}-\frac{1}{P r}\left(\frac{\partial T}{\partial x}+S_{M} \frac{\partial C}{\partial x}\right)=0 & \text { at } z=1, \\
u=w=\frac{\partial T}{\partial x}=\frac{\partial(C-T)}{\partial x}=0 & \text { at } x=0 \text { and } x=A
\end{array}
$$

\subsection{Infinite layer}

For a horizontally infinite layer, we replace the boundary condition $(4.2 d)$ at the lateral walls by periodic boundary conditions of imposed wavenumber $k$. We seek steady bifurcations, i.e. $\lambda=0$.

After the following change of variables (Hurle \& Jakeman 1971, Bergeon et al. 1994a):

$$
\begin{array}{ll}
C^{\prime}=\frac{C-T}{S c}, & T^{\prime}=\frac{T}{P r}, \\
\boldsymbol{U}^{\prime}=M a \boldsymbol{U}, & p^{\prime}=M a p
\end{array}
$$

equations (4.1)-(4.2c) become

$$
\begin{aligned}
& 0=\nabla^{2} \boldsymbol{U}^{\prime}-\nabla p^{\prime}, \\
& 0=-w^{\prime}+\nabla^{2} T^{\prime}, \\
& 0=-w^{\prime}+\nabla^{2} C^{\prime}, \\
& 0=\nabla \cdot \boldsymbol{U}^{\prime},
\end{aligned}
$$

with boundary conditions

$$
\begin{array}{ll}
u^{\prime}=w^{\prime}=T^{\prime}=\frac{\partial C^{\prime}}{\partial z}=0 & \text { at } z=0, \\
w^{\prime}=\frac{\partial T^{\prime}}{\partial z}=\frac{\partial C^{\prime}}{\partial z}=0 & \text { at } z=1, \\
\frac{\partial u^{\prime}}{\partial z}-M a\left(1+S_{M}\right)\left(\frac{\partial T^{\prime}}{\partial x}+\frac{S c}{P r} \frac{S_{M}}{1+S_{M}} \frac{\partial C^{\prime}}{\partial x}\right)=0 & \text { at } z=1 .
\end{array}
$$

This formulation makes it clear that the linear stability problem depends only on two combinations of the four parameters $M a, S_{M}, P r, S c$, which we choose as

$$
\overline{M a} \equiv M a\left(1+S_{M}\right) \quad \text { and } \quad \psi_{M} \equiv \frac{S c}{\operatorname{Pr}} \frac{S_{M}}{1+S_{M}} .
$$




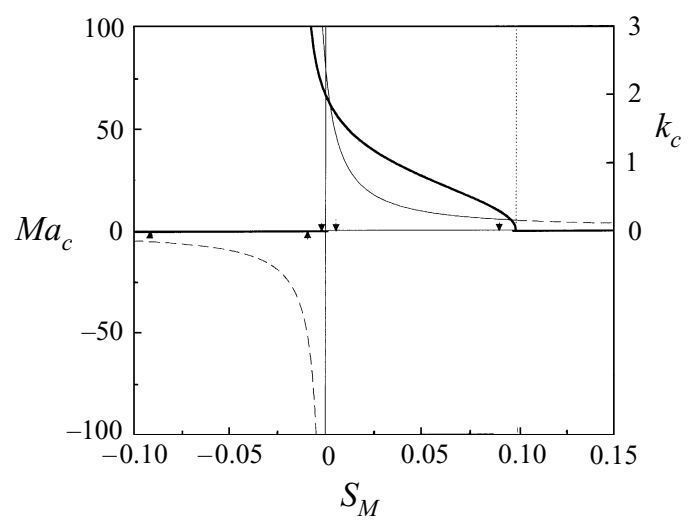

FIGURE 3. Linear stability analysis for steady bifurcations in an infinite horizontal domain for $\operatorname{Pr}=0.6, S c=60$. Heavy solid curves: critical wavenumber $k_{c}$ as a function of $S_{M}$. For $S_{M}<0$, upper (lower) curve represents $M a>0(M a<0)$. Note that $k_{c}=0$ for $S_{M}<0, M a<0$ and for $S_{M}>0.0989$, called the solutal-dominated regime. Thin curves: critical Marangoni number $M a_{c}$ as a function of $S_{M}$. The thin dashed curve is the relation $M a_{c} S_{M}=48 \mathrm{Pr} / \mathrm{Sc}$ obeyed by the thresholds in the solutal-dominated regime. The thin solid curve represents the thresholds outside of the solutal-dominated regime, delimited by the dotted line at $S_{M}=0.0989$. Triangles represent bifurcation diagrams to be presented in later sections.

Upon substituting a horizontal dependence $\mathrm{e}^{\mathrm{i} k x}$, a system is obtained which can be solved analytically in terms of hyperbolic functions in $z$. Each value of $\psi_{M}$ and $k$ yields a value of $\overline{M a}$; minimization over $k$ yields the critical values $k_{c}$ and $\overline{M a}_{c}$ for each $\psi_{M}$.

The key point found by Bergeon et al. 1995 is that the critical wavenumber $k_{c}$ is zero for $\psi_{M}>9$ and for $\psi_{M}<0$. In the limit as $k \rightarrow 0, w^{\prime}$ and $T^{\prime}$ vanish, while $u^{\prime}$ and $C^{\prime}$ remain finite. The first and third terms in equation $(4.5 c)$ balance one another, and there remains only one parameter $\psi_{M} \overline{M a}=M a S_{M} S c / P r$, whose critical value is found to be 48 . We call this the solutal-dominated regime.

To facilitate comparison with the remainder of the article, we translate to the usual parameters $M a$ and $S_{M}$. Figure 3 shows $k_{c}$ and $M a_{c}$ as a function of $S_{M}$ for our values of $P r=0.6, S c=60$. For $S_{M}>0.0989, M a>0$ and for $S_{M}<0, M a<0$ we have $k_{c}=0$ and $M a_{c} S_{M}=48 \mathrm{Pr} / S c=0.48$ This is the solutal-dominated regime. For $S_{M}<0.0989, M a>0$, the critical wavenumber $k_{c}$ is finite. Note that this domain contains both positive and negative values of $S_{M}$. However, for $S_{M}<0, M a>0$, the steady-state bifurcation is preceded by a Hopf bifurcation at lower $M a$; this regime will be discussed in $\$ 4.3 .2$.

Figure 3 is qualitatively similar to that calculated for Rayleigh-Bénard convection in binary mixtures, originally by Hurle \& Jakeman (1971) and by Nield (1964), and subsequently by many other authors (see Barten et al. 1995).

The triangles in figure 3 represent detailed cases that will be studied in this paper. Bifurcation diagrams will be presented for the values of $S_{M}$ designated by the triangles. Triangles located above and below the abscissa represent positive or negative values of $M a$, respectively.

\subsection{Pure fluid}

We next consider the stability problem of Marangoni convection of a pure fluid in a finite container. Setting $S_{M}=0$ decouples $(\boldsymbol{U}, T)$ from the concentration field $C$. We 


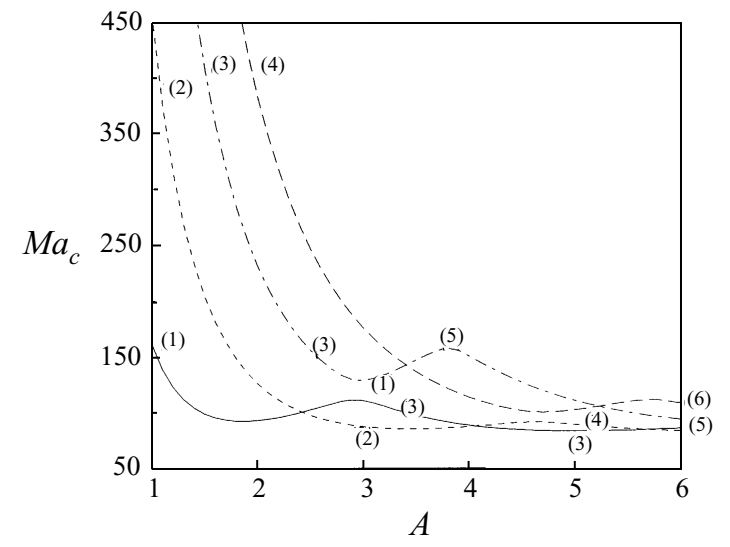

FiguRE 4. Critical Marangoni numbers for first four bifurcations in a pure fluid as a function of aspect ratio. Integers indicate the number of rolls of the bifurcating eigenvector. Solid and dash-dotted curves indicate bifurcations to an odd number of rolls. Short-dashed and long-dashed curves indicate bifurcations to an even number of rolls. Note that the number of rolls (but not the parity) changes continuously along a curve.

continue to seek steady bifurcations $\lambda=0$. Equations (4.1)-(4.2) then become

$$
\begin{aligned}
& 0=\nabla^{2} \boldsymbol{U}-\nabla p, \\
& 0=-M a w+\frac{1}{P r} \nabla^{2} T, \\
& 0=\nabla \cdot \boldsymbol{U},
\end{aligned}
$$

with boundary conditions

$$
\begin{array}{ll}
u=w=T=0 & \text { at } z=0, \\
\frac{\partial u}{\partial z}-\frac{1}{\operatorname{Pr}} \frac{\partial T}{\partial x}=w=\frac{\partial T}{\partial z}=0 & \text { at } z=1, \\
u=w=\frac{\partial T}{\partial x}=0 & \text { at } x=0 \text { and } x=A .
\end{array}
$$

Solutions to (4.7)-(4.8) are found using the technique described in $\S 3.4$.

Figure 4 displays the dependence of the first four bifurcation points on aspect ratio. (Similar figures are shown by Winters et al. 1988 and by Dijkstra 1992.) As we shall see explicitly in $\S 5$, only the first (lowest $M a_{c}$ ) bifurcation leads directly to a stable flow. Subsequent (higher $M a_{c}$ ) bifurcations are responsible for the creation of branches which are initially unstable, but which may be stabilized at yet higher $M a$. From the analysis presented in $\S 4.1$, we know that the limit of $M a_{c}$ for infinite aspect ratio is 80 , a result first established by Pearson (1958).

Many of the features of figure 4 result from the symmetry of the equations and boundary conditions under $x$-reflection, i.e. reflection through $x=A / 2$. All eigenvectors are either symmetric or antisymmetric in $x$. Symmetric eigenvectors contain an even number of cells whereas antisymmetric eigenvectors contain an odd number of cells. As a consequence of the horizontal reflection symmetry, the number of rolls on a bifurcation curve remains either odd or even and cannot change parity. Curves representing eigenvectors of opposite parity cross at codimension-two bifurcation points; those of the same parity do not. Figure 4 clearly shows the phenomenon of avoided crossings, whereby same-parity curves approach one another 

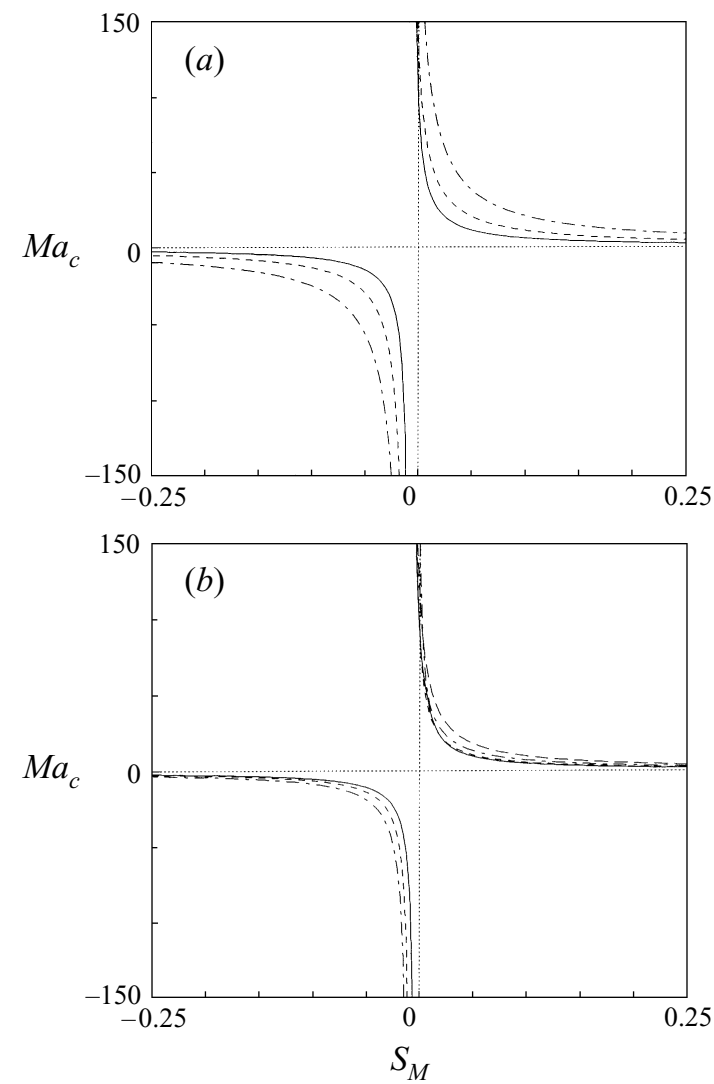

FIGURE 5. Critical Marangoni numbers for steady bifurcations from the conductive state as a function of Soret coefficient for $A=2(a)$ and $A=4(b)$. In (a), solid, dashed, and long-and-short-dashed curves correspond to one-roll, two-roll, and three-roll structures, respectively. Additional long-dashed curve in $(b)$ corresponds to four-roll structure.

and then recede. Near their point of closest approach, the eigenvectors along the curves exchange qualitative attributes - in this case, simply the number of rolls. In figure 4 , this means that the number of rolls, indicated along the curves, changes via the creation and annihilation of rolls which are initially infinitesimally small. As argued by Winters (Cliffe \& Winters 1986, Winters et al. 1988, Riley \& Winters 1989) and others (e.g. Hirschberg \& Knobloch 1996) this is characteristic of convection problems with no-slip lateral boundaries. The situation is very different when the lateral boundaries are free-slip, as in the study of Rosenblat et al. (1982) and Dauby et al. (1993). In the free-slip case, each mode possesses a different translational symmetry, which allows any paths to cross (Riley \& Winters 1989).

\subsection{Binary mixture}

We now turn to the steady coupled thermal-solutal problem, i.e. the solution of equations (4.1)-(4.2) with $\lambda=0$. Figure 5 depicts the dependence of the critical Marangoni numbers $M a_{c}$ on the Soret coefficient $S_{M}$. We plot critical Marangoni numbers corresponding to the first three or four bifurcations for two different aspect ratios: $A=2$ and $A=4$.

We see that $\left|M a_{c}\right|$ decreases with $\left|S_{M}\right|$ for each bifurcation. This is because the destabilizing solutal contribution to the surface force increases with $\left|S_{M}\right|$. The linear 


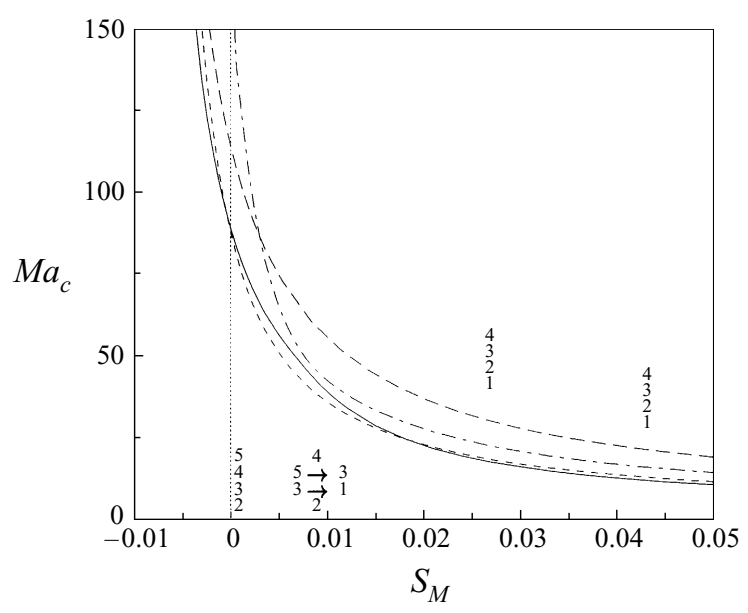

FiguRE 6. Enlargement of figure $5(b)$ for $S_{M} \approx 0$. Number of rolls corresponding to each of the four curves is indicated by four stacked integers. These change when curves cross or when the solution evolves along a curve.

stability analysis for the infinite domain (Bergeon et al. 1995) has shown that the dependence of critical Marangoni number $M a$ on wavenumber $k$ becomes increasingly flat as $S_{M}$ is increased. In the finite domain, this effect is seen as the accumulation of the curves associated with the different bifurcations as $S_{M}$ is increased; successive bifurcations to structures with different numbers of rolls occur at nearly the same value of $M a$.

In most of the parameter range studied, the bifurcations encountered in order of increasing $M a_{c}$ are to one-roll, two-roll, and three-roll structures. This sequence is not respected for $A=4,0<S_{M}<0.018$ (figure 6). In this range, bifurcation curves cross, for the following reason: As $S_{M}$ is increased from 0 , the critical wavenumber in an infinite domain decreases from $k_{c}=2$ to $k_{c}=0$. For $A=4$, this gives a favoured configuration of $8 / \pi=2.5$ rolls for $S_{M}=0$ and one large roll for $S_{M}$ large. A change in the number of rolls should take place approximately when $k_{c}=1.5 \pi / A=1.2$, which is near $S_{M}=0.018$. In addition, the number of rolls (but not the parity) corresponding to a given curve changes continuously with $S_{M}$. Thus, the solid curve in figure 6 corresponds to a three-roll structure for $S_{M} \leqslant 0$ which evolves continuously to a oneroll structure as $S_{M}$ is increased. This bifurcation has the lowest critical Marangoni number when $S_{M} \leqslant 0$, but in the small range $0<S_{M}<0.018$, delimited by two crossings of curves, bifurcation to a two-roll structure occurs for lower $M a_{c}$.

\subsubsection{Solutal-dominated regime}

The existence of a solutal-dominated regime in horizontally infinite layers motivates us to define a solutal-dominated regime for cavities of finite aspect ratio as well. We recall from $\S 4.1$ that in the infinite layer, the temperature and vertical velocity of the critical mode vanish when the critical wavenumber becomes zero. The flow is then driven exclusively by the solutal contribution to the surface tension. The criterion of zero wavenumber clearly cannot be satisfied in cavities of finite aspect ratio. We can, however, seek circumstances under which the thermal contribution to the surface tension is small and dominated by the solutal contribution.

Our goal is to derive a pure solutal problem analogous to the pure thermal problem of $\S 5.1$. Returning to system (4.4)-(4.5), we see that it is possible to decouple $\left(\boldsymbol{U}^{\prime}, C^{\prime}\right)$ 
from $T^{\prime}$ if we can neglect the thermal contribution to the surface tension in the boundary condition (4.5c). This is achieved for $\left|\psi_{M}\right|$ large if $C^{\prime}$ is approximately of the same order as, or larger than, $T^{\prime}$. Equations $(4.4 b, c)$ imply that $C^{\prime}$ and $T^{\prime}$ are indeed of the same order. Thus, in the limit of large $\left|\psi_{M}\right|$, system (4.4)-(4.5) reduces to one coupling only $\boldsymbol{U}^{\prime}$ and $C^{\prime}$.

If we now wish to rewrite the $\left(U^{\prime}, C^{\prime}\right)$ system in terms of the original variables, we see from the definitions $(4.3 a)$ of $C^{\prime}$ and $T^{\prime}$ that if $C^{\prime} \sim T^{\prime}$, then $C \sim(1+S c / P r) T$. If we make the additional hypothesis that $S c / P r$ is large, as in our case where $S c / P r=100$, then $C \gg T$ so that in fact $C^{\prime} \approx C / S c$. Using both of these approximations, i.e. using $\left|\psi_{M}\right| \gg 1$ to justify neglecting the thermal contribution to the surface tension and using $S c / P r \gg 1$ to justify $C \gg T$, we rewrite system (4.4)-(4.5) in the original variables, and obtain what we shall call the pure solutal problem:

$$
\begin{aligned}
& 0=\nabla^{2} \boldsymbol{U}-\nabla p, \\
& 0=-M a w+\frac{1}{S c} \nabla^{2} C, \\
& 0=\nabla \cdot \boldsymbol{U},
\end{aligned}
$$

with boundary conditions

$$
\begin{array}{ll}
u=w=\frac{\partial C}{\partial z}=0 & \text { at } z=0, \\
\frac{\partial u}{\partial z}-\frac{S_{M}}{\operatorname{Pr}} \frac{\partial C}{\partial x}=w=\frac{\partial C}{\partial z}=0 & \text { at } z=1, \\
u=w=\frac{\partial C}{\partial x}=0 & \text { at } x=0 \text { and } x=A .
\end{array}
$$

The interpretation of the pure solutal problem (4.9)-(4.10) is more problematic than that of the pure thermal problem. Since concentration gradients are established by the Soret effect, the thermal gradient is necessary in order to establish the concentration gradient of the conductive state. However, we can then consider the pure solutal problem as one in which the temperature field is 'frozen' to its conductive profile as a consequence of $S c \gg P r$. Perturbations of $T$ are damped far more rapidly than those in $C-T$. Thus perturbations of $T$ will not become neutrally stable; instead, neutrally stable modes of (4.1)-(4.2) will consist primarily of perturbations of $C-T$, i.e. of $C$. The requirement $\left|\psi_{M}\right|$ large serves to ensure that whatever small component of $T$ is present in the mode contributes negligibly to the surface tension.

We shall define the solutal-dominated regime for the finite-size domain as the regime over which the thermal-solutal problem can be well approximated by the pure solutal problem. In practice, if we require $\left|\psi_{M}\right|>9$, by analogy with the infinite layer, then the solutal-dominated regime for $S c / P r=100$ consists of the ranges $S_{M}>0.0989$ and $S_{M}<-0.0826$.

System (4.9)-(4.10) remains a valid approximation even when $S c / P r$ is not large, as long as $\left|\psi_{M}\right|$ is large and $C$ is replaced by $C-T$. But in this case, its significance is much more restrictive. Rather than implying that the concentration perturbations drive the instability, the condition $\left|\psi_{M}\right| \gg 1$ requires $S_{M}$ near -1 , i.e. a coincidence of parameters governing the Soret effect and surface tension; see (2.3). Again requiring $\left|\psi_{M}\right|>$ 9, then (4.9)-(4.10) for $(\boldsymbol{U}, C-T)$ holds for $S c / P r=1$ if $-1.125<S_{M}<-0.9$. Such cases will not be included in our definition of the solutal-dominated regime.

We solve equations (4.9)-(4.10) numerically by the technique described in $\S 3.4$. It is easily seen that the solutal problem depends only on the single parameter $M a S_{M} S c / P r$ 


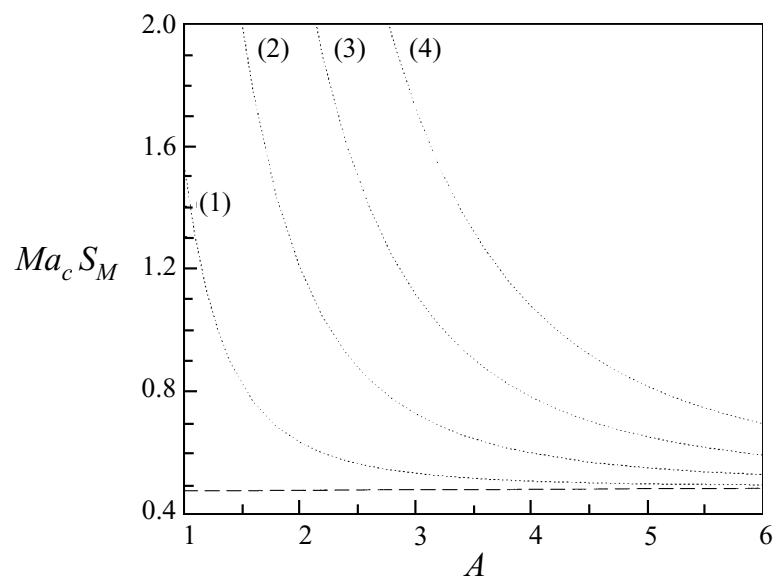

FiguRE 7. $M a_{c} S_{M}$ for the pure solutal problem as a function of aspect ratio $A$ for $S c / P r=100$. Integers indicate the number of rolls associated with each bifurcation curve. Dashed line is the relation $M a_{c} S_{M}=48 \mathrm{Pr} / S c$ obtained analytically for the solutal-dominated regime in a horizontally infinite cavity.

(i.e. $\overline{M a} \psi_{M}$ in the variables of (4.6)), along with the aspect ratio $A$. Fixing $S c / P r=100$, the product $M a_{c} S_{M}$ at the convective threshold is a function of $A$. In figure 7 we plot the dependence of $M a_{c} S_{M}$ on $A$ for the first four bifurcations, corresponding to one-, two-, three- and four-roll flows. All four curves decrease monotonically with $A$ and approach the limiting value $M a_{c} S_{M}=48 \mathrm{Pr} / S c=0.48$ derived in $\S 4.1$ for a horizontally infinite layer. The curves do not cross.

Figure 7 highlights a crucial difference between the pure solutal and the pure thermal problem, whose thresholds are shown in figure 4 . Because the preferred thermal configuration consists of slightly elongated rolls $\left(k_{c}=2\right.$ for infinite aspect ratio) the number of rolls increases with $A$. The thresholds oscillate as $A$ is increased through multiples of the preferred roll size. In contrast, the preferred solutal configuration is the widest possible roll ( $k_{c}=0$ for infinite aspect ratio). The first bifurcation is always to a one-roll structure. This leads to a monotonic decrease of the threshold with $A$ as the roll widens and the preferred size is approached.

We now compare the pure solutal thresholds with those of the thermal-solutal problem in figure 8 . The aspect ratio is $A=4$, for which thresholds have already been shown in figure $5(b)$, and $S c / P r=100$. Positive values of $S_{M}, M a$ are shown in figure $8(a)$; negative $S_{M}, M a$ values are shown in figure $8(b)$. Note that the thermal-solutal thresholds for $S_{M}, M a$ positive (negative) are lower (higher) than the pure solutal thresholds, because the thermal contribution is destabilizing (stabilizing).

We see that the pure solutal and the thermal-solutal thresholds are quite close together in the range shown, i.e. $\left|S_{M}\right|>0.05$, especially for the first bifurcation. For $S_{M}=0.09$ and $A=4$, a case which we will study in detail in $\S 5$ and which we shall include within the solutal-dominated regime, the first bifurcation of the thermalsolutal problem occurs at $M a=5.44$, whereas the threshold for the pure solutal problem is $M a=5.62$, a difference of only about $3 \%$.

\subsubsection{Stabilizing solutal contribution: oscillation}

We now turn to the case in which a stabilizing solutal contribution counteracts a destabilizing thermal contribution. It is well known that the first bifurcation is a 


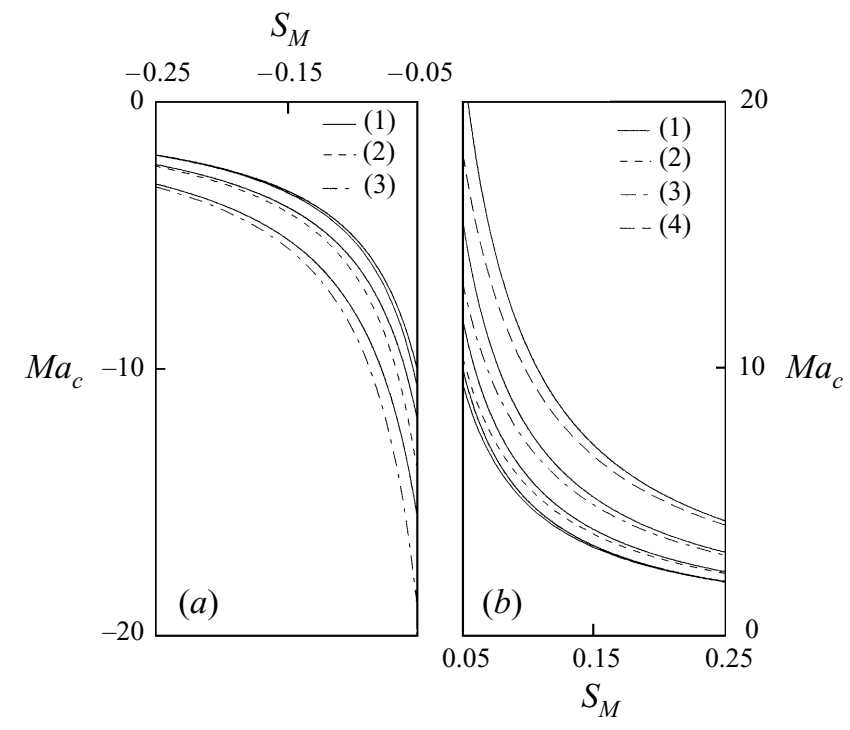

FIGURE 8. Comparison of thresholds $M a_{c}$ for the pure solutal problem and for the thermal-solutal problem as a function of $S_{M}$ for $A=4$ and $S c / P r=100$. Thermal-solutal thresholds (labelled by number of rolls) are those shown in figure $5(b)$, pure solutal thresholds (nearby solid curves) are computed from values on figure 7. Thermal contribution neglected in pure solutal problem leads to higher thermal-solutal thresholds for $S_{M}$ negative $(a)$ and to lower $\left|M a_{c}\right|$ thermal-solutal thresholds for $S_{M}$ positive $(b)$. Agreement is best for the first bifurcation. Solutal-dominated regime defined as range of $S_{M}$ for which the two thresholds differ by less than $5 \%$.

Hopf bifurcation if $S_{M}$ is sufficiently negative. (The value of $S_{M}$ below which this is true depends on $S c / P r$, but in our case of a liquid with large $S c / P r$, it is very close to zero.) Hopf bifurcation points, at which the eigenvalues have zero real part but non-zero imaginary part, are found by calculating the leading eigenvalues $\lambda$ of the problem (4.1)-(4.2) by the method described in section 3.5.

Figure $9(a)$ displays the critical Marangoni numbers for the first two Hopf bifurcations $H$ and $H^{\prime}$ and the first three steady bifurcations, to one-, two-, and three-roll flows, for aspect ratio $A=2$. Note that the critical Marangoni number for each Hopf bifurcation is almost independent of $S_{M}\left(M a_{H}\right.$ varies only between 94 and 96 in the range of $S_{M}$ shown), whereas that for the steady bifurcations varies greatly. Steady bifurcation curves corresponding to different numbers of rolls cross, as was already seen in figure 6 for $A=4$. Each of the two Hopf bifurcation curves calculated terminates at a small negative value of $S_{M}$ on a steady bifurcation curve with a similar spatial structure. That is, the complex conjugate pair of eigenvectors belonging to the first Hopf bifurcation $H$ are antisymmetric in $x$ and contain a single roll; those belonging to $H^{\prime}$ are symmetric and contain two rolls.

The frequency at the first Hopf bifurcation is depicted in the inset on figure $9(b)$. The frequency decreases to zero with $S_{M}$, or equivalently, the period diverges. This is a trademark of the disappearance of the Hopf bifurcation at a codimension-two point. We note that Rayleigh-Bénard convection with Soret effect also features a codimension-two point at small negative $S_{M}$; see e.g. Brand, Hohenberg \& Steinberg (1984), Knobloch (1986). Knobloch \& Moore (1988) have remarked that in an infinite layer, the wavenumbers of the steady and Hopf bifurcations are different, so that the codimension-two point is absent. Thus, the infinite period characterizing 

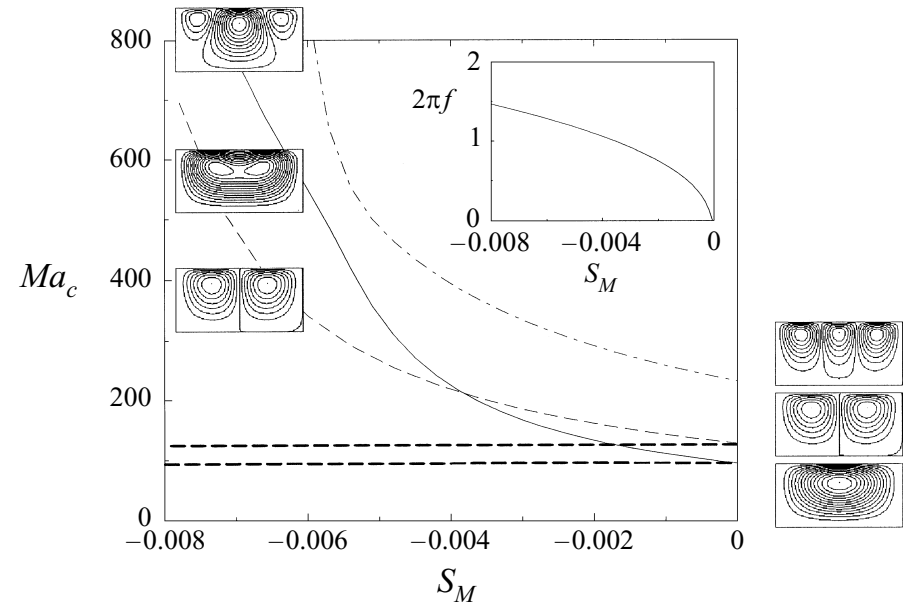

FIGURE 9. Critical Marangoni numbers for steady and Hopf bifurcations for negative $S_{M}$ and $A=2$. Solid, dashed, and long-and-short dashed curves indicate steady bifurcations to one-, two-, and three-roll flows, as seen in the corresponding streamfunction contours. Heavy dashed lines indicate Hopf bifurcations. Both Hopf bifurcation curves terminate by joining steady bifurcation curves at very small negative values of $S_{M}$. Inset: frequency $f$ (times $2 \pi$ ) of first Hopf bifurcation.

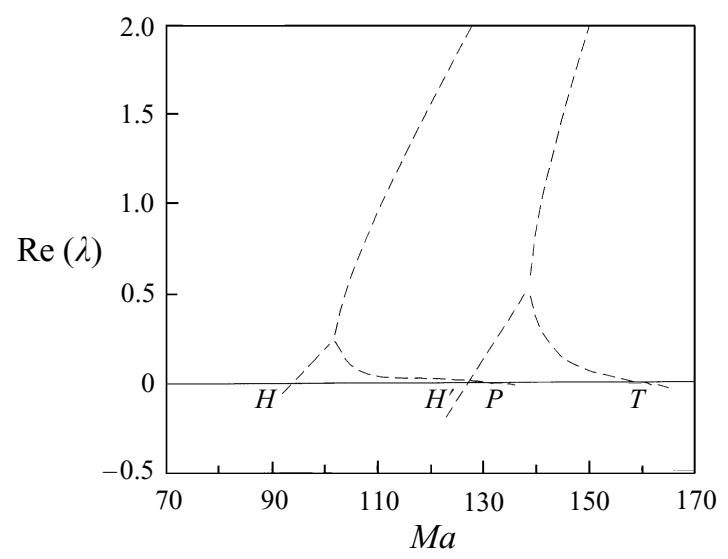

Figure 10. Real part of most unstable eigenvalues as a function of $M a$ for $S_{M}=-0.002, A=2$ Hopf bifurcations $H$ and $H^{\prime}$ occur at $M a_{H}=94.01$ and $M a_{H^{\prime}}=128.5$, steady bifucations $P$ and $T$ at $M a_{P}=132.4$ and $M a_{T}=160.0$.

a codimension-two point can be observed only in a sufficiently small aspect ratio container, where the spatial structure of the flow is constrained.

Figure 10 depicts the intricate interlacing between the real and complex unstable eigenvalues. The real part of the most unstable eigenvalues is shown as a function of Marangoni number for the values $A=2, S_{M}=-0.002$. After crossing zero at the Hopf bifurcation point $H$, the unstable complex conjugate pair splits into two real eigenvalues. One of these continues to increase with $M a$, while the other decreases, vanishing at a steady bifurcation $P$ to a one-roll state. The second complex conjugate pair of eigenvalues undergoes the same process, with the decreasing real eigenvalue crossing zero at a bifurcation $T$ to a two-roll state. 


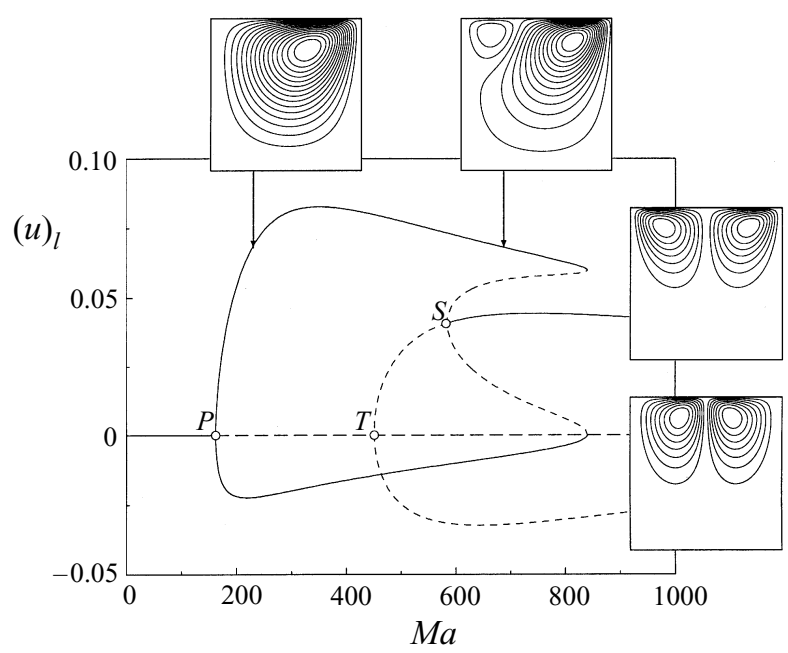

FigURE 11. Bifurcation diagram for a pure fluid $\left(S_{M}=0\right)$ with $A=1$. Insets are streamfunction contours. $P, T$, and $S$ indicate primary pitchfork, transcritical, and secondary pitchfork bifurcations at $M a_{P}=161.7, M a_{T}=450.6, M a_{S}=581.6$, respectively. Solid curves indicate stable states, long-dashed line the unstable portion of the conductive branch, and short-dashed curves unstable convective states. Resolution is $13 \times 13$.

\section{Nonlinear study}

We present bifurcation diagrams for Marangoni convection, first in a pure fluid $\left(S_{M}=0\right)$, and then in a binary mixture with Soret effect, for various combinations of stabilizing and destabilizing thermal and solutal contributions. Many of the features of the diagrams are dictated by the symmetry of the problem (e.g. Crawford \& Knobloch 1991). Flows with an even number of rolls are invariant under horizontal reflection: a bifurcation to such a flow is transcritical. Flows with an odd number of rolls occur in pairs: the corresponding bifurcation is a pitchfork. The variable $(u)_{l}$ plotted along the ordinate of our bifurcation diagrams is the vertical velocity at a fixed spatial location. For each diagram, the location is chosen to best portray the features of the bifurcation diagram; a typical choice is $x=A / 8, z=3 / 4$. This projection has the important advantage of allowing both pitchfork and transcritical bifurcations to be represented on the same figure (a global quantity such as the total kinetic energy or maximum velocity would not distinguish between two asymmetric flows related by $x$-reflection). Note that pitchfork bifurcations do not appear symmetric in this representation and that the diagrams contain intersections which are not bifurcations but merely coincident values of $(u)_{l}$ at the chosen spatial location. Finally, we recall that velocity has been non-dimensionalized by $M a v / H$. Thus the nearly constant value to which $(u)_{l}$ tends with $M a$ in some cases when the bifurcating branches are well-established means that the velocity in conventional units actually increases linearly with $M a$.

\subsection{Pure fluid}

We have computed the bifurcation diagrams for integer aspect ratios varying from $A=1$ to $A=4$.

The bifurcation diagram for $A=1$ presented in figure 11 (for $\mathrm{Pr}=0.6$ ) is qualitatively identical to that of figure 2 (for $\operatorname{Pr}=8$ ). The first primary bifurcation is a pitchfork $P$ to a one-roll structure, while the second one is a transcritical bifurcation $T$ to a two-roll structure. Since the primary steady bifurcation points are independent 


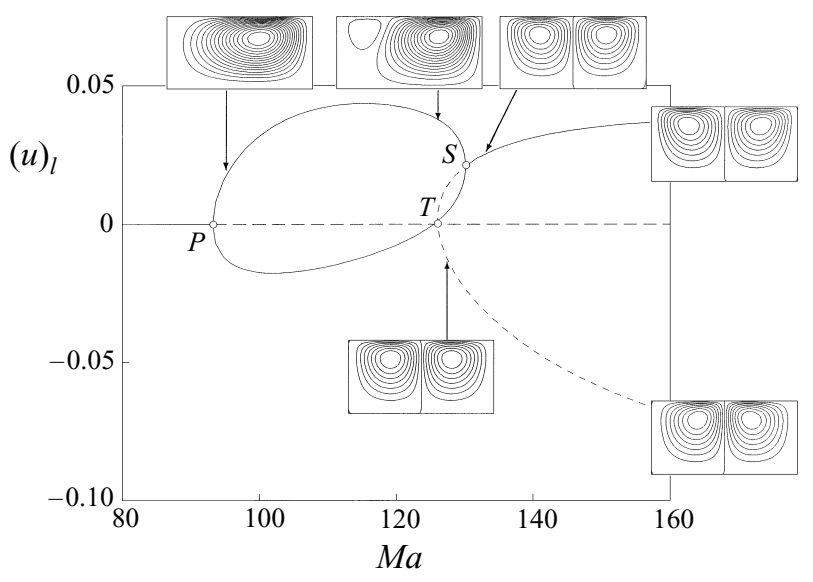

FIGURE 12. Bifurcation diagram for a pure fluid with $A=2$. Insets are streamfunction contours. $P, T$, and $S$ indicate primary pitchfork, transcritical, and secondary pitchfork bifurcations at $M a_{P}=93.76, M a_{T}=126.08, M a_{S}=130.3$ respectively. Solid curves indicate stable states, long-dashed line the unstable portion of the conductive branch, and short-dashed curves unstable convective states. Resolution is $15 \times 13$.

of Prandtl number, the threshold values are equal to those obtained with $\operatorname{Pr}=8$ (figure 2).

For a fixed Marangoni number, the two solutions along the branches resulting from the pitchfork bifurcation $P$ are dynamically equivalent: the solutions on one branch are obtained from those on the other by reflection in $x$. One consequence is that both branches undergo saddle-node bifurcations at the same Marangoni number. With increasing $M a$, a counter-rotating roll grows in the corner at which the velocity is upwards. As we continue to follow each branch around its saddle-node bifurcation, the new roll continues to grow. Eventually, at one value of $M a$, the new and original rolls are of exactly the same size and shape; the flow is reflection-symmetric in $x$. At this point, our two branches meet a third branch and disappear via a secondary subcritical pitchfork bifurcation, labelled $S$.

This third branch, containing two-roll states with up-flow at the centre ('up-flowing branch'), originates from the transcritical bifurcation. Although it branches leftwards towards $M a<M a_{T}$ at onset, it quickly undergoes a saddle-node bifurcation and reverses direction. The range of hysteresis between the transcritical and saddle-node bifurcations is too small to be visible on the figure. Another branch of two-roll states with down-flow at the centre ('down-flowing branch') also emerges supercritically from $T$. Since $T$ occurs after $P$, in order of increasing $M a$, both branches of two-roll states are unstable at onset. The up-flowing branch is stabilized by the secondary pitchfork bifurcation $S$; for higher $M a$, it is the only stable branch on this figure.

This type of interaction between symmetric and asymmetric states via primary pitchfork, transcritical, and secondary pitchfork bifurcations $P, T$, and $S$ will reoccur throughout our study. The hysteresis associated with transcritical bifurcations remains too small to be seen in any of the bifurcation diagrams we will present, and we shall not refer to it in discussing these diagrams.

The results for the aspect ratio $A=2$ are presented in figure 12 . The only qualitative difference with the previous $A=1$ case is that the asymmetric branches do not undergo saddle-node bifurcations, and so the secondary pitchfork bifurcation at which they terminate is supercritical. 


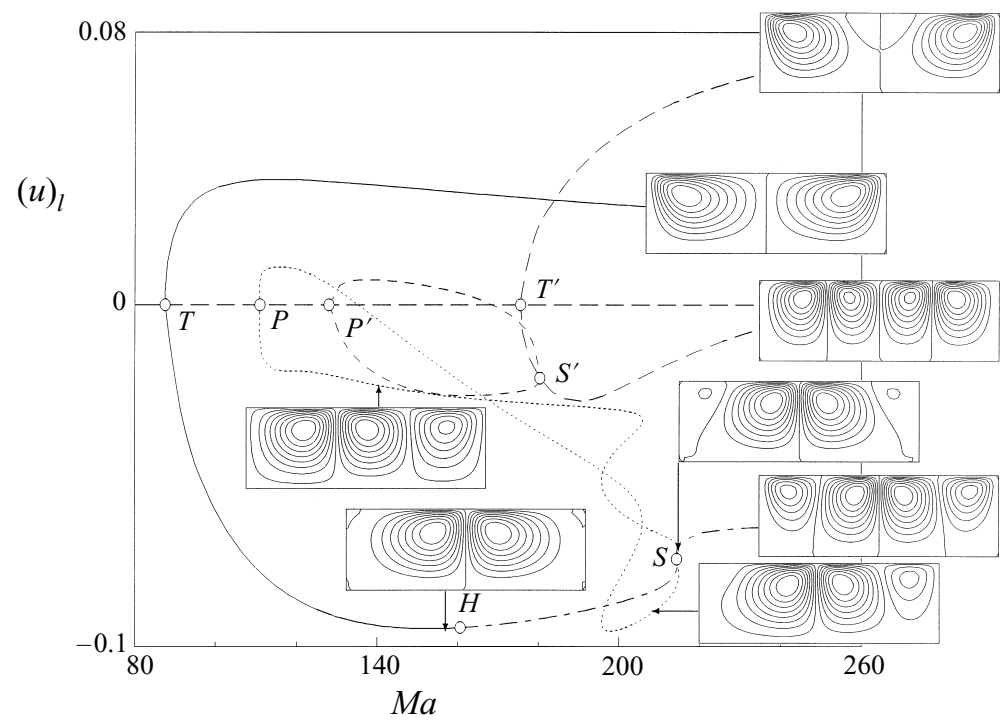

FIGURE 13. Bifurcation diagram for a pure fluid and $A=3$. Insets are streamfunction contours. $P, P^{\prime}$ and $T, T^{\prime}$ indicate primary pitchfork and transcritical bifurcations, respectively, at $M a_{T}=87.5, M a_{P}=110.9, M a_{P^{\prime}}=128.0$, and $M a_{T^{\prime}}=175.6 . S, S^{\prime}$ and $H$ indicate secondary pitchfork and Hopf bifurcations, respectively, with $M a_{H}=162.0$. Solid curves represent stable conductive or two-roll states. Dotted and short-dashed curves are branches containing unstable three- and one-roll states. Long-dashed curves represent unstable four-roll states and the unstable portion of the conductive branch. The dashed-and-dotted curve represents a portion of the down-flowing two-roll branch which has been destabilized by $H$. Each three-roll branch undergoes three saddle-node bifurcations before terminating on $S$. Resolution is $21 \times 13$.

For $A=3$ (figure 13), the order of the primary bifurcations is reversed: $T$ precedes $P$, as can also be seen on figure 4. The pitchfork bifurcation $P$ is now slightly subcritical and associated with three-roll structures. The three-roll branches emerging from $P$ each undergo three successive saddle-node bifurcations, giving the bifurcation diagram a complicated appearance. However, the basic scenario is as before: these branches terminate at a secondary pitchfork bifurcation $S$ with a two-roll branch emerging from $T$.

In this case, $A=3$, it is the down-flowing supercritical two-roll branch that is involved in, and destabilized by $S$. Along this branch occurs a secondary Hopf bifurcation (labelled $H$ ). This bifurcation is probably subcritical, since time-dependent simulations slightly above $H$ display growing oscillations that terminate on the steady up-flowing two-roll branch. Another occurrence of the basic scenario is seen in figure 13 , whereby branches of one-roll states emerging from $P^{\prime}$ annihilate at $S^{\prime}$ with a branch of four-roll states emerging from $T^{\prime}$ (see also figure 4). All of the branches emerging from $P^{\prime}$ or $T^{\prime}$ remain unstable. Just as for the lower aspect ratios, it is the up-flowing two-roll branch that remains as the sole stable steady state at the highest end of our Marangoni number range.

The bifurcation diagram for $A=4$ is presented in figure 14. As in the case $A=3$, a transcritical bifurcation to two-roll flows is followed by a pitchfork bifurcation to three-roll flows. Here, these are very closely spaced in $M a$ (see also figure 4). Because the secondary pitchfork destabilizes the branch of down-flowing two-roll states, for $M a>M a_{S}$, only the up-flowing branch is stable. The up-flowing branch is in turn destabilized by another secondary pitchfork bifurcation $S^{\prime}$. Each of the branches of 
(a)

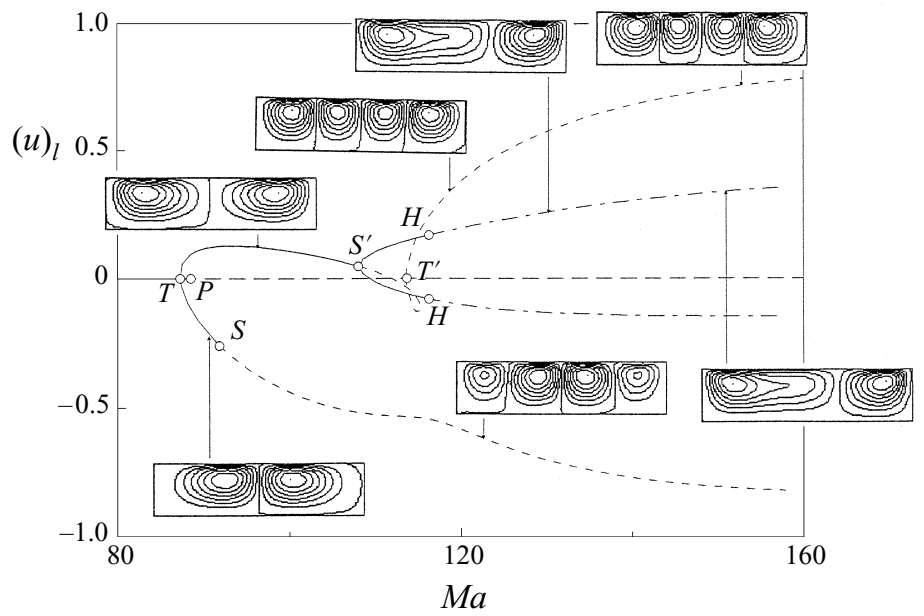

(b)

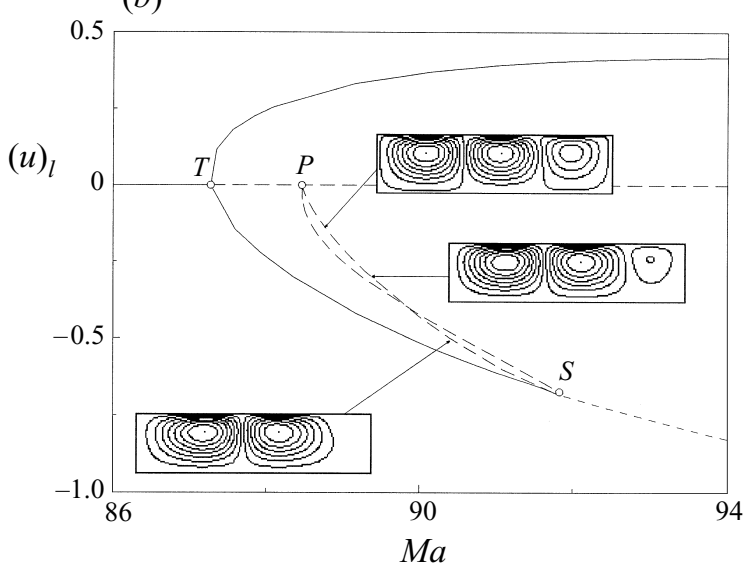

FiguRE 14. (a) Bifurcation diagram for a pure fluid and $A=4$. Insets are streamfunction contours. $P$ and $T, T^{\prime}$ indicate primary pitchfork and transcritical bifurcations, respectively, at $M a_{T}=87.26$, $M a_{P}=88.46$, and $M a_{T^{\prime}}=113.68 . S, S^{\prime}$ and $H$ indicate secondary pitchfork and Hopf bifurcations, respectively, at $M a_{S}=91.8, M a_{S^{\prime}}=108.16, M a_{H}=116.27$. Solid curves represent stable conductive, two-roll, and asymmetric two-roll branches. Short-dashed curves represent unstable four-roll branches. Long-dashed curve represents unstable portion of the conductive branch. Long-and-short-dashed curve represents asymmetric up-flowing two-roll branches which have been destabilized by Hopf bifurcations $H$. (b) Enlargement of $(a)$. Transcritical bifurcation $T$ to two-roll branches is closely followed by pitchfork bifurcation $P$ to three-roll branches. These are destroyed at a secondary pitchfork bifurcation $S$ which destabilizes the down-flowing two-roll branch. Resolution is $21 \times 13$.

asymmetric two-roll states created is subsequently destabilized by a Hopf bifurcation. The resulting stable oscillatory flow has been described in Bergeon et al. (1994a). Both the down-flowing and up-flowing two-roll branches evolve into four-roll states as $M a$ is increased. For the down-flowing branch, this evolution is straightforward. For the up-flowing branch, this process is rather tortuous: after acquiring two additional small rolls at the centre, the branch undergoes a saddle-node bifurcation and then intersects the conductive branch a second time in another transcritical bifurcation $T^{\prime}$. 


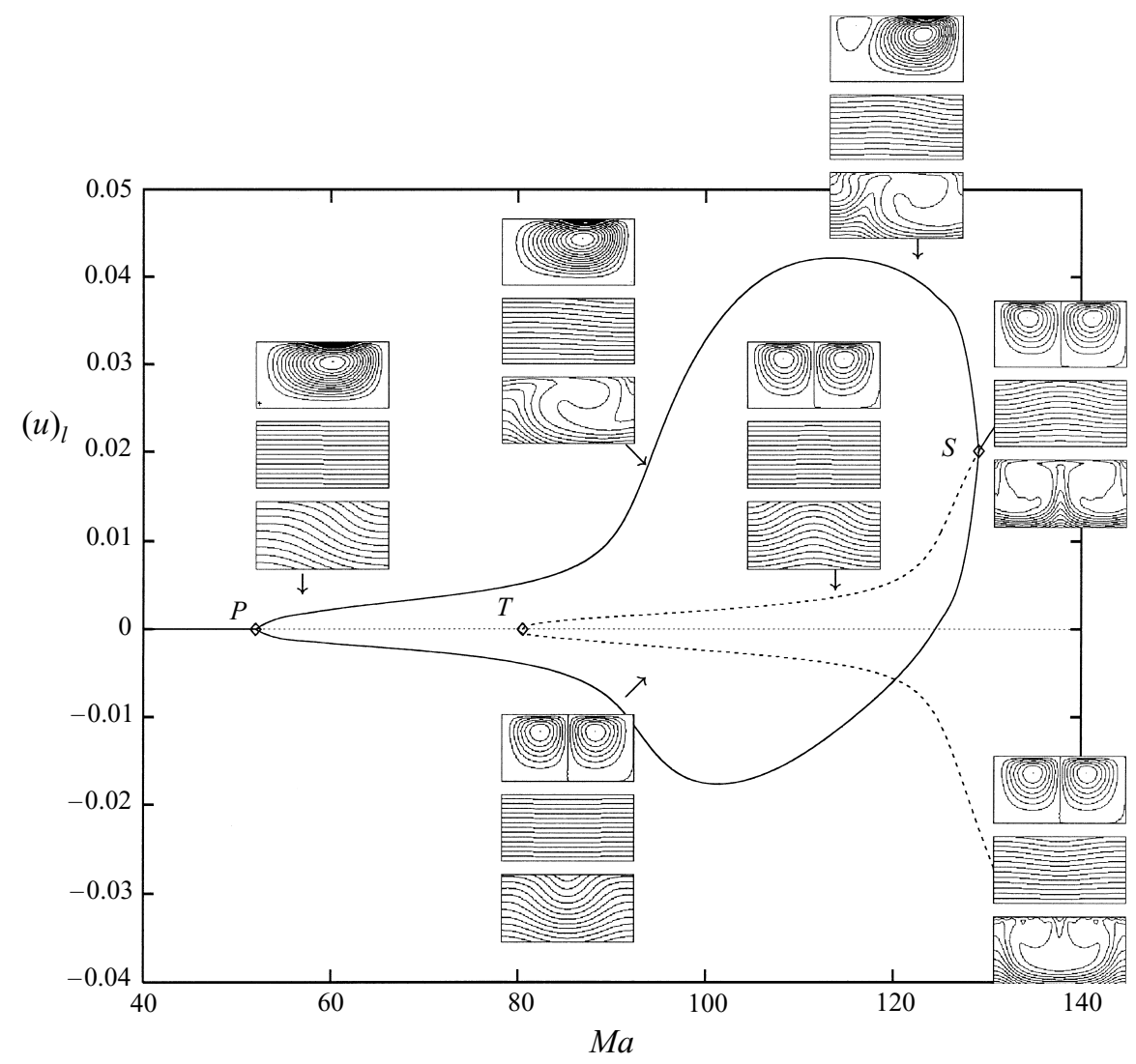

FIGURE 15. Bifurcation diagram for $S_{M}=0.0054, A=2$. Insets show streamfunction, temperature, and concentration contours. $P, T$, and $S$ indicate primary pitchfork, transcritical, and secondary pitchfork bifurcations, respectively, at $M a_{P}=51.96, M a_{T}=80.46$, and $M a_{S}=129.0$. Note the change in slope near $M a=94$, the threshold for pure thermal convection. Resolution is $15 \times 13$.

(We have confirmed this intersection by verifying that the four rolls reverse direction of rotation as the branch crosses the axis.)

\subsection{Binary mixture}

We will describe representative scenarios from different quadrants of the $\left(M a, S_{M}\right)$ parameter plane (see figure 3). These correspond to stabilizing or destabilizing thermal and solutal contributions.

\subsection{1. $S_{M}>0$ and $M a>0$ : Soret and Marangoni regimes}

In this quadrant, both thermal and solutal contributions are destabilizing. We will present two cases, one with a small solutal contribution $\left(S_{M}=0.0054\right)$ and one in the solutal-dominated regime $\left(S_{M}=0.09\right)$.

For $S_{M}=0.0054$ and aspect ratio $A=2$, the bifurcation diagram depicted in figure 15 contains the same basic features as figure 12 for a pure fluid: a pitchfork, a transcritical, and a secondary pitchfork bifurcation. The bifurcation thresholds for the binary fluid are considerably below those for the pure fluid; between the two thresholds, the vertical velocity component plotted along the ordinate varies slowly and remains small. Near the pure fluid threshold $M a \approx 94$, the slopes of the bifurcating branches change abruptly. In figure 16 we superpose the two diagrams 


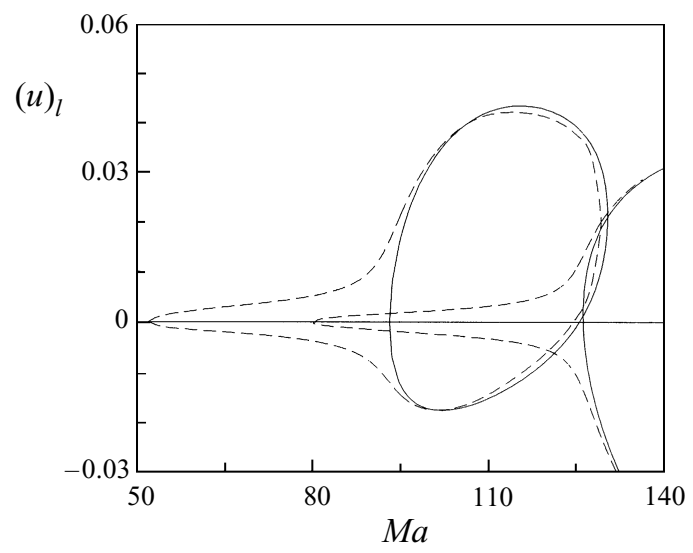

FigURE 16. Superposition of bifurcation diagrams for $S_{M}=0.0054$ (dashed curves) and $S_{M}=0$ (solid curves) for $A=2$. The thresholds for $S_{M}=0.0054$ are considerably lower than the pure thermal thresholds. However, above the pure thermal thresholds, the branches from the two diagrams almost coincide.

and observe that, above the bifurcation thresholds for the pure fluid, the branches for the pure and binary fluid coincide quite well.

In order to explain this, we examine the structure of the velocity, temperature, and concentration fields along each branch in figure 15. The concentration fields are far more distorted by the flow than are the temperature fields because the large ratio $S c / P r=100$ indicates that temperature diffuses much faster than concentration.

We can distinguish two regimes. Just above the convective threshold, the velocity is small. The temperature gradients $\partial T / \partial x$ at the free surface are much smaller than the concentration gradients $\partial C / \partial x$, but the contributions $\partial T / \partial x$ and $S_{M} \partial C / \partial x$ to the driving term in $(2.5 c)$ are of the same order. For example, in figure 15 , for $M a=60$, the maximum values along the surface are $(\partial T / \partial x)_{\max }=0.005,(\partial C / \partial x)_{\max }=0.5$, and $S_{M}(\partial C / \partial x)_{\max }=0.003$. A weakly convecting regime also exists in buoyancy-driven convection (Barten et al. 1995). There, it is called the Soret regime, and we will adopt this nomenclature for the Marangoni case as well.

When the thermal threshold is reached, a much stronger convective motion is triggered by the now-unstable vertical temperature gradient. Much greater mixing of the solute ensues. The concentration field is nearly uniform over much of the container, with boundary layers and plumes whose spatial resolution is the most demanding feature of these computations. The contribution $\partial T / \partial x$ to the driving term in $(2.5 c)$ is now much larger than $S_{M} \partial C / \partial x$. For example, in figure 15, for the one-roll flow at $M a=100$, the maximum values along the surface are $(\partial T / \partial x)_{\max }=0.1$, $(\partial C / \partial x)_{\max }=0.75$, and $\left(S_{M} \partial C / \partial x\right)_{\max }=0.004$. We will call this the Marangoni regime, by analogy to the strongly convecting Rayleigh regime (Barten et al. 1995) seen in buoyancy-driven convection.

Note that the Soret regime is defined for a given $S_{M}$ to be the portion of the nonlinear convective branch for $M a$ between the thermal-solutal and the pure thermal thresholds, where convection is weak. In contrast, the solutal-dominated regime denotes a range of $S_{M}$ such that the linear threshold of the thermal-solutal problem $M a_{c}$ is given approximately by that of the pure solutal problem of $\S 4.3 .1$.

We now turn to a case within the solutal-dominated regime, $S_{M}=0.09$ and aspect ratio $A=4$. The bifurcation diagram shown in figure 17 is qualitatively very similar 


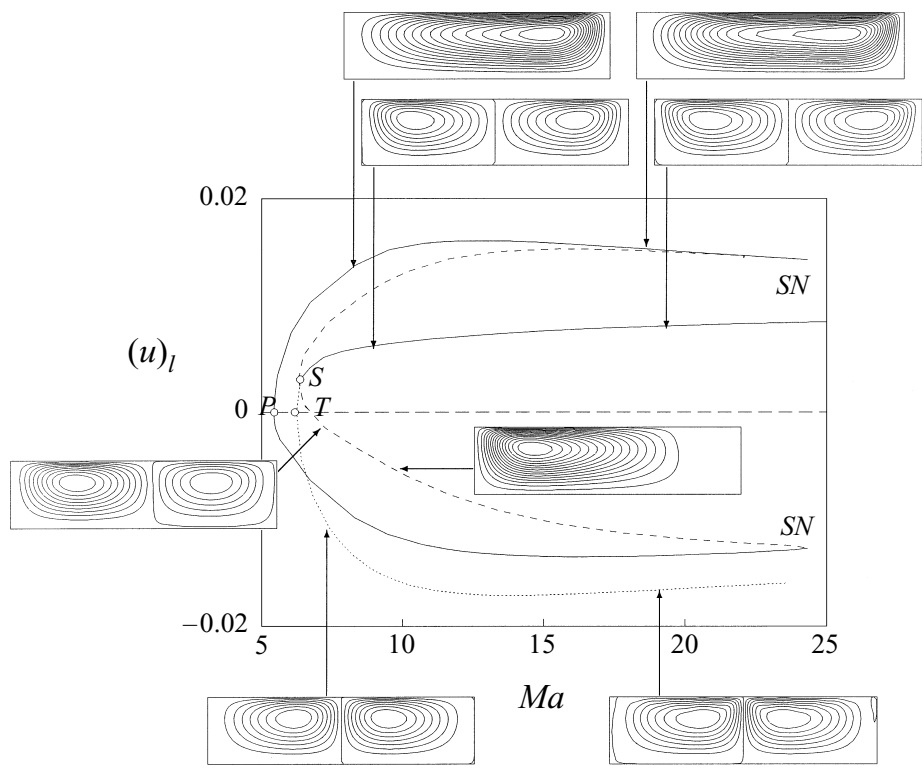

FiguRE 17. Bifurcation diagram for $S_{M}=0.09, A=4$ in the solutal-dominated regime. Insets are streamfunction contours. Primary bifurcations are pitchfork $(P)$ to one-roll states (solid curve) at $M a_{P}=5.44$, and transcritical bifurcation $(T)$ to two-roll states (dotted curve) at $M a_{T}=6.16$. Secondary subcritical pitchfork $(S)$ at $M a_{S}=6.36$ stabilizes up-flowing two-roll branch (solid curve). Dashed curves denote asymmetric unstable convective states. Saddle-node bifurcations occur at $M a_{S N}=24.3$. Resolution is $29 \times 15$.

to those previously discussed. The flow is driven primarily by the solutal contribution to the surface force. In contrast to the pure fluid case of figure 14 for the same aspect ratio, the first bifurcation $P$ here is to a one-roll state. This was already seen in figure 5 and is expected from the results of $\S \S 4.1$ and 4.3.1. The next bifurcations, $T$, which creates a pair of two-roll branches, and $S$, which stabilizes the up-flowing two-roll branch, occur very close in $M a$. As a consequence, there is bistability over most of the range of existence of the one-roll branches. Finally, a pair of saddle-node bifurcations destroys the one-roll states, leaving the up-flowing two-roll state as the only stable steady state. From figure 5 it can be seen that other primary bifurcations occur in the range of values of figure 17; we have not computed the unstable branches resulting from these subsequent bifurcations.

As discussed previously for the case $S_{M}=0.0054$, we expect the flow intensity to increase sharply and the solution to resemble that obtained for a pure fluid when the Marangoni number exceeds the pure thermal threshold of $M a=87$ (not shown in figure 17), i.e. when we pass from the Soret to the Marangoni regime. We have verified that this is indeed the case.

\subsection{2. $S_{M}<0$ and $M a<0$ : stabilizing thermal gradient}

We now investigate pattern selection when a destabilizing solutal force is counteracted by a stabilizing thermal force. In this quadrant of the $\left(M a, S_{M}\right)$ parameter plane, all convective states belong to the Soret regime, since pure thermal convection cannot occur. The first instability is always to a one-roll structure (see figure 5).

Figure 18 depicts the bifurcation diagram for $S_{M}=-0.09$ and $A=4$, a case in the solutal-dominated regime. This diagram is qualitatively similar to that obtained for $S_{M}=0.09$ and $M a>0$; in particular, three stable solutions exist over a large range 


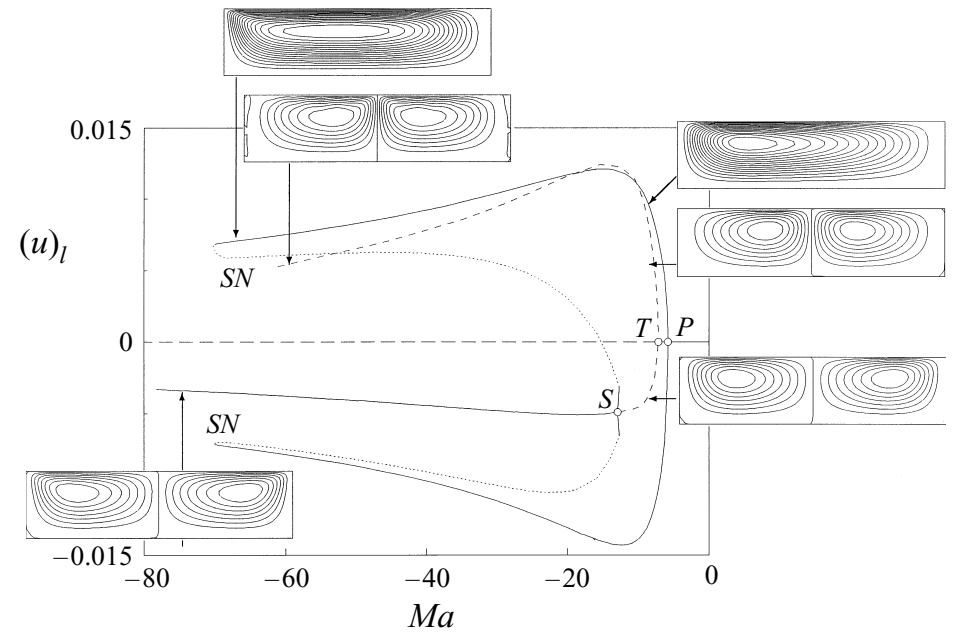

FIGURE 18. Bifurcation diagram for $S_{M}=-0.09, A=4$ in the solutal-dominated regime. Insets are streamfunction contours. Primary bifurcations are pitchfork $P$ to one-roll states (solid curve) at $M a_{P}=-5.81$, and transcritical bifurcation $T$ to two-roll states (short-dashed curve) at $M a_{T}=-7.18$. Secondary subcritical pitchfork $(S)$ at $M a_{S}=-12.71$ stabilizes up-flowing two-roll branch (solid curve). Saddle-node bifurcations occur at $M a_{S N}=-69.98$. Dotted curves denote asymmetric unstable convective states. Resolution is $29 \times 15$.

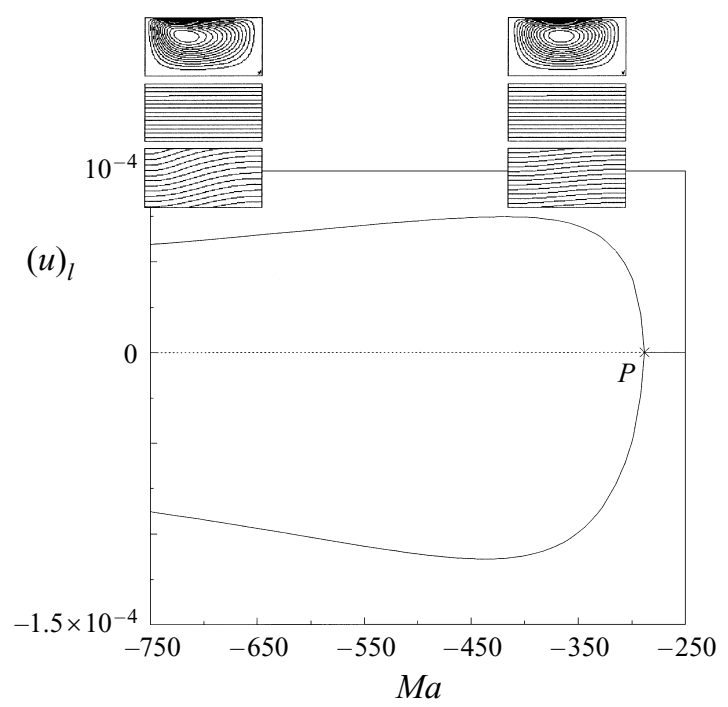

Figure 19. Bifurcation diagram for $S_{M}=-0.009, A=2$. Pitchfork bifurcation $P$ at $M a=-288$ creates a pair of one-roll states. Resolution is $29 \times 15$.

of Marangoni number. The absolute values of the thresholds $\left|M a_{P}\right|,\left|M a_{T}\right|$ are larger for $S_{M}=-0.09$ than for $S_{M}=0.09$ because the thermal contribution is stabilizing.

The bifurcation diagram for a smaller $\left|S_{M}\right|\left(S_{M}=-0.009\right)$ is presented in figure 19 for $A=2$. This case is well outside the solutal-dominated regime: the critical Marangoni number for the pure solutal problem can be calculated from figure 7 to be $M a_{c} \approx-70$, whereas the actual thermal-solutal threshold is seen from figure 19 to be $M a=-288$ due to the stabilizing contribution of the temperature. The one-roll branches created at this pitchfork bifurcation remain stable beyond $M a=-800$, 


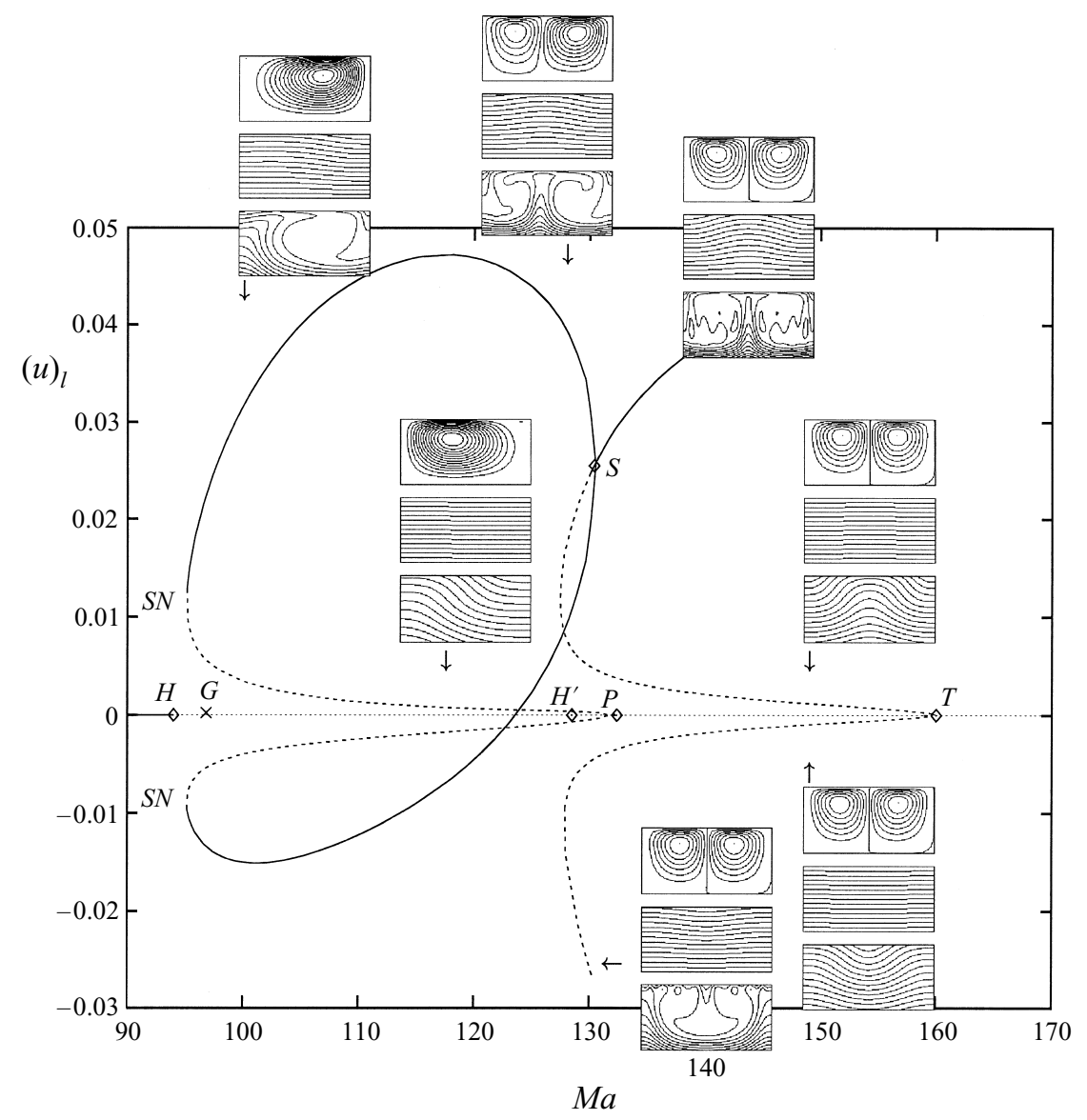

FIGURE 20. Bifurcation diagram for $S_{M}=-0.002, A=2$. Insets are contours of streamfunction, temperature, and concentration fields along the branches indicated by arrows. Primary bifurcations in order of increasing $M a$ are two supercritical Hopf bifurcations $H$ at $M a_{H}=94.01$ and $H^{\prime}$ at $M a_{H^{\prime}} \approx 128.5$ to limit cycles consisting primarily of one-roll and two-roll states, respectively, followed by pitchfork and transcritical bifurcations $P$ and $T$ at $M a_{P}=132.4$ and $M a_{T}=160$ to pairs of one- and two-roll states, respectively. Also on this diagram are a pair of saddle-node bifurcations $\left(M a_{S N}=95.16\right)$ stabilizing the one-roll branches created at $P$, a global bifurcation $\mathrm{G}\left(M a_{G} \approx 98\right)$ in which the limit cycle created at $H$ is destroyed in a collision with the unstable one-roll steady states, and a secondary pitchfork bifurcation $S\left(M a_{S}=130.4\right)$ which terminates the pair of one-roll steady states and stabilizes the upflowing two-roll steady state. Resolution is $21 \times 15$.

and they are also the only solutions in this range. The flow remains weak and the concentration unmixed despite the high values of $|M a|$; this is typical of the Soret regime. The temperature gradients $\partial T / \partial x$ are much smaller than those of the concentration $\partial C / \partial x$, as was the case in the Soret regime for $S_{M}=0.0054$. Here, for $S_{M}=-0.009$, their contributions to the surface tension (equation $(2.5 c)$ ) are approximately equal and opposite: For example, at $M a=-300,(\partial T / \partial x)_{\max }=$ $5 \times 10^{-4},(\partial C / \partial x)_{\max }=8 \times 10^{-2}$, and $S_{M}(\partial C / \partial x)_{\max }=-7 \times 10^{-4}$.

\subsection{3. $S_{M}<0$ and $M a>0$ : oscillations}

We have investigated the effect of a small stabilizing solutal contribution $\left(S_{M}=\right.$ -0.002 ) for aspect ratio $A=2$. We recall that linear stability analysis of the conductive solution shows that the first and second primary bifurcations are Hopf bifurcations; 
(a)
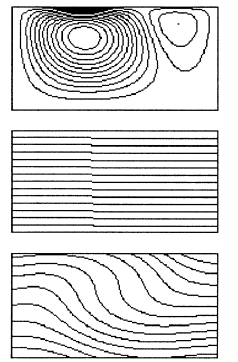

(b)
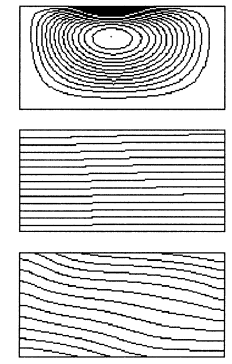

(c)
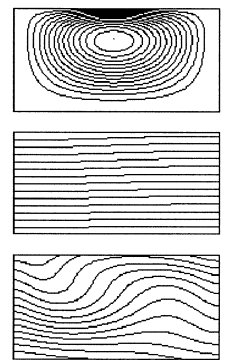

(d)
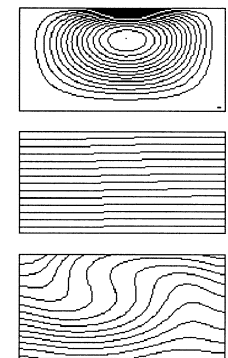

Figure 21. Limit cycle at $M a=95, S_{M}=-0.002$, and $A=2$. Only half a period is included; the other half is obtained by reflection in $x$. Contours of the streamfunction (top), temperature (middle), and concentration (bottom) fields are shown. The first snapshot $(a)$ shows a large clockwise-turning roll being invaded by a new small counter-clockwise roll. In the subsequent three snapshots $(b-d)$, the counter-clockwise roll occupies the entire cavity and distorts the concentration contours. The temperature field is less distorted and reacts much faster to changes in the velocity field. In the next half-period, the counter-clockwise roll will in turn be destroyed by a new clockwise roll emerging at the left boundary.
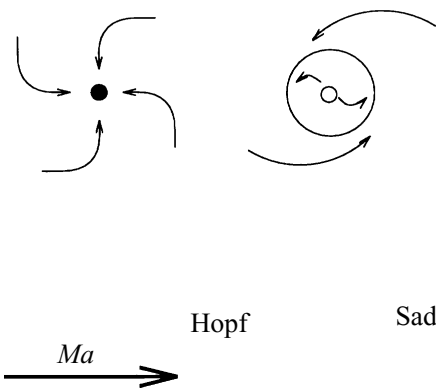

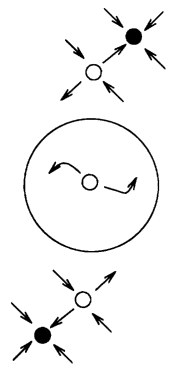

Saddle-node
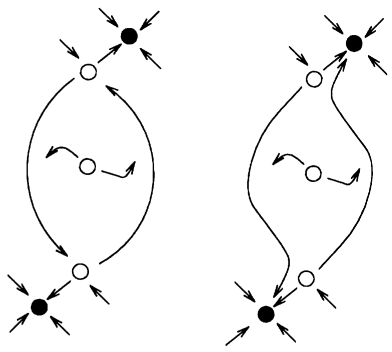

Heteroclinic (global)

FIGURE 22. Phase portraits associated with the first bifurcations of figure 20 for $S_{M}=-0.002$. Hopf bifurcation destabilizes the conductive state and gives rise to a limit cycle shown in figure 21 . Saddle-node bifurcations then form pairs of symmetrically related stable and unstable steady states (saddles). At the global bifurcation, the limit cycle includes the two saddles and has infinite period. For higher $M a$, the limit cycle no longer exists, and trajectories converge to one of the two stable steady states.

see figure 9. The bifurcation diagram is depicted in figure 20. The first Hopf bifurcation $H$ at $M a_{H}=93.95$ is supercritical. The resulting limit cycle is shown for $M a=95$ in figure 21. A cycle consists of two long phases, each resembling a one-roll steady state, separated by two short phases in which a small counter-rotating roll appears in the upper down-flowing corner, and grows quickly, replacing the large roll. A physical interpretation of a similar periodic flow is given in Bergeon et al. (1994a).

This limit cycle is closely related to the steady one-roll solutions, which appear at a pair of saddle-node bifurcations at $M a_{S N}=95.16$. It is by colliding with the unstable one-roll solutions that the limit cycle disappears. This process is depicted via schematic phase portraits in figure 22 . As $M a$ is increased from its value $M a_{H} \approx 94$ at the Hopf bifurcation, the limit cycle spends an increasing amount of time in the vicinity of each of the one-roll solutions, until, at a value $M a_{G} \approx 98$, a global saddleconnection bifurcation occurs. At this value, the limit cycle is a heteroclinic orbit, i.e. 
it contains both one-roll solutions and is of infinite period. For higher values of $M a$ the limit cycle no longer exists; transition will take place to one of the one-roll states. This transition is slightly hysteretic, occurring at $M a_{G} \approx 98$ for increasing $M a$ and at $M a_{S N} \approx 95$ for decreasing $M a$. The range $94 \leqslant M a \leqslant 98$ over which the limit cycle exists is itself quite small.

We turn now to the steady states, which follow a variant of the usual scenario. Oneroll branches emerge from the conductive branch via a primary pitchfork bifurcation $P$ at $M a_{P}=132.4$. This bifurcation differs from those seen in the $S_{M} \geqslant 0$ cases in that the one-roll solutions branch towards $M a<M a_{P}$. (The bifurcation is nevertheless supercritical because the critical eigenvalue increases in the direction of branching: it is negative for $M a>M a_{P}$ and positive for $M a<M a_{P}$, as shown in figure 10. This feature was also observed by DaCosta, Knobloch \& Weiss (1981) in buoyancy-driven double-diffusive convection.) Two-roll branches emerge at a transcritical bifurcation $T$ at $M a_{T}=160$, in a similar fashion. Except for the very small interval between $T$ and its accompanying saddle-node bifurcation (not visible on the figure), both the two-roll solutions branch towards values $M a<M a_{T}$; see figures 9,10 and 20 . ( $T$ is preceded by another Hopf bifurcation $H^{\prime}$, much as $P$ is preceded by $H$, which gives rise to an oscillation between two-roll states. Because this limit cycle is unstable, we have not investigated it and shall not discuss it further, except to conjecture that it too terminates via a global bifurcation.) The unstable one-roll branches are stabilized at the saddle-node bifurcations $S N$ and terminate at a secondary pitchfork bifurcation at $M a_{S}=130.4$, which in turn stabilizes the upflowing two-roll branch. The sequence of stable solutions observed as $M a$ is increased is thus: limit cycle alternating between one-roll states; either of the one-roll states; up-flowing two-roll state.

This bifurcation scenario is widely observed in buoyancy-driven convection in binary fluids, e.g. by Platten \& Chavepeyer $(1973,1975)$ for Rayleigh-Bénard convection with Soret effect, and by DaCosta et al. (1981) for double-diffusive convection. In these particular cases, saddle-node bifurcations precede the Hopf bifurcation in control parameter ( $R a$ rather than $M a$ ). This means that, while the transition sequence with increasing control parameter is similar to that we have described, the oscillatory regime is bypassed when the control parameter is decreased.

In order to deepen our understanding of the $S_{M}=-0.002$ case, we carry out two comparisons with other values of $S_{M}$. In figure 23, we superpose the steady solutions with those obtained for a pure fluid, as was previously done in figure 16 for $S_{M}>0$. The prominent saddle-node bifurcations in the $S_{M}=-0.002$ diagram are quite close to the pure thermal thresholds, and the stable portions of the one- and two-roll branches closely coincide with their pure thermal analogues. The difference between the $S_{M}<0$ and the pure thermal case is the much higher thresholds for the primary steady bifurcations, accompanied by the extensive leftward-branching unstable portions of the one- and two-roll branches, We can extend our previous definition of the Soret regime to include these unstable weakly convective states existing between the pure thermal threshold and the thermal-solutal threshold. The Marangoni regime is extended to include the stable convective states created by the saddle-node bifurcations.

Figure 24 compares the real part of the leading eigenvalues as a function of $M a$ for three values of $S_{M}: S_{M}=-0.002, S_{M}=0$ and $S_{M}=0.002$. Based on this diagram, we can classify the eigenvalues as solutally or thermally dominated. For $S_{M}=0.002$, the leading eigenvalue undergoes a sudden change in slope at the pure thermal threshold $M a \approx 94$. This eigenvalue can be considered as solutally dominated for $M a<94$, where it is small, and thermally dominated for $M a>94$, where it approaches closely 


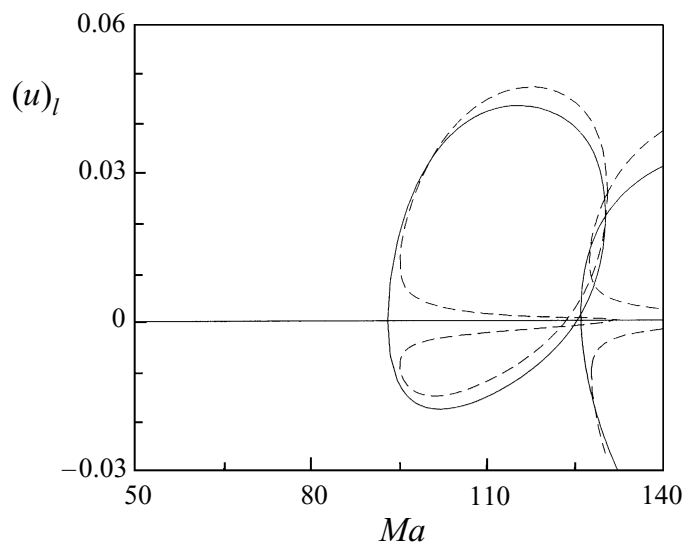

FiguRE 23. Superposition of bifurcation diagrams for $S_{M}=-0.002$ (dashed curves) and $S_{M}=0$ (solid curves) for $A=2$. Despite the important qualitative differences between the two bifurcation diagrams, the branches of steady states are remarkably close, except for the leftward-branching steady states of $S_{M}=-0.002$.

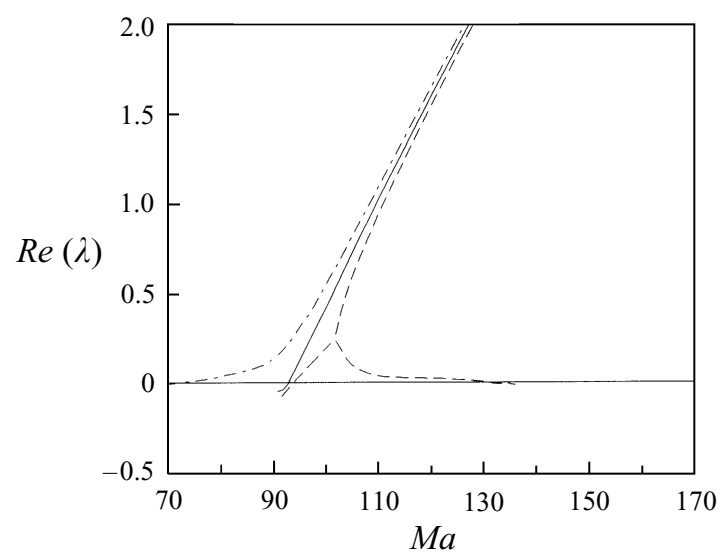

FiguRE 24. Real part of the most unstable eigenvalues for $A=2$ as a function of $M a$ for $S_{M}=0.002$ (dotted-and-dashed curve), $S_{M}=0$ (solid curve) and $S_{M}=-0.002$ (dashed curves). For $S_{M}=-0.002$, the complex conjugate pair divides into two real eigenvalues at $M a=101.2$. Portions of eigenvalue curves approaching the $S_{M}=0$ eigenvalue curve, i.e. $S_{M}=0.002, M a>90$ and upper curve for $S_{M}=-0.002, M a>101$, are associated primarily with the thermal problem, eigenvalue curves with smaller slope, i.e. $S_{M}=0.002, M a<90$ and lower curve for $S_{M}=0.002, M a>101$, are associated primarily with the solutal problem. Complex conjugate pair is mixed solutal-thermal.

the leading eigenvalue for the pure thermal problem. For $S_{M}=-0.002$, the situation is more complicated. The complex conjugate pair of eigenvalues which are the first to bifurcate can be considered to be a mixed solutal-thermal pair. When these split into two real eigenvalues, the larger one rises towards the pure thermal eigenvalue and the smaller one decreases towards zero and can be considered as solutally dominated. The relative magnitude of the thermally dominated eigenvalues is determined by $S_{M}$ : for the same value of $M a$, the eigenvalue is larger (smaller) when the solutal contribution is destabilizing (stabilizing).

Finally, we mention that we have also studied cases with more negative $S_{M}$. For $S_{M}=-0.006$, similar results are obtained, i.e. a supercritical Hopf bifurcation leading to an oscillatory one-roll flow and leftward branching primary bifurcations 
to steady states. The unstable solutal branches and their connection with the steady bifurcation points are more complex since, for this value of $S_{M}$, the steady primary bifurcation points are interchanged: the transcritical bifurcation to two-roll states precedes the pitchfork bifurcation to one-roll states (see figure 6). For $S_{M}=-3$, $M a=100$, and $A=4$, we find oscillations throughout which the flow retains reflection symmetry in $x$. The configuration alternates between two and four rolls, as cells are periodically created and annihilated at the centre and at the sidewalls; see Bergeon et al. (1994a). We have not conducted a detailed study, but a few simulations indicate that oscillations take place over a larger range in $M a$. This is to be expected when we are farther from the codimension-two point at which the frequency of the Hopf bifurcation is zero.

\section{Conclusions}

Our numerical investigation of Marangoni convection with Soret effect has yielded a number of general conclusions. As in the case of Rayleigh-Bénard convection, linear stability analysis demonstrates the existence of a solutal-dominated regime in a horizontally infinite domain for which the critical Marangoni number for steady convection is inversely proportional to $S_{M}$ and the critical wavenumber is zero. Qualitatively, it is clear that in a finite domain, a zero wavenumber corresponds to a single large roll and that thresholds are larger since the pattern must adapt to the container. Quantitatively, we have been able to extend the applicability of the solutaldominated regime to a finite domain by defining and solving a pure solutal problem which is the asymptotic limit of the thermal-solutal (Marangoni-Soret) problem for large $S c / P r$ and $\left|S_{M}\right|$ not too small.

We have calculated bifurcation diagrams both for pure fluids and for binary fluids with Soret effect, for aspect ratios ranging from $A=1$ to $A=4$ and various values of $S_{M}$. Although the details are specific to each case, the bifurcation diagrams do share a number of general features.

In most of the cases we have studied, the initial bifurcation is to a one-roll state, even outside the solutal-dominated regime. As $M a$ is increased, the one-roll states are succeeded by a two-roll state with upflow in the centre of the cell. This occurs via an interaction between the one- and two-roll branches, specifically a secondary bifurcation which annihilates the one-roll branches and stabilizes the upflowing tworoll branch. Within this broad framework, there may or may not be bistability between the one- and the two-roll branches, depending on whether the bifurcations are superor subcritical.

The favoured outcome of an upflowing two-roll state highlights an important difference between our case and that of Rayleigh-Bénard convection governed by the Boussinesq equations. The Boussinesq approximation guarantees reflection symmetry in $z$ as well as in $x$. As a consequence, all primary bifurcations (to a single layer of rolls) are pitchforks. The bifurcation scenario linking our one- and upflowing two-roll branches is ruled out because up-flow and down-flow are strictly equivalent. While we do not expect one- and two-roll branches to play as important a role in larger aspect ratios, we do expect similar bifurcation scenarios involving interplay between branches arising from successive pitchfork and transcritical bifurcations.

In figure 25 , we summarize the ways in which the bifurcation diagram for thermal convection in a pure fluid (figures $25 b$ and 12 ) is modified by inclusion of the Soret effect.

For small positive $S_{M}$ (figures $25 c$ and 15) we distinguish two distinct convective 
(a)
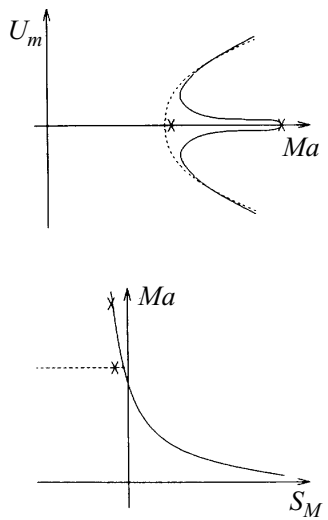

(b)
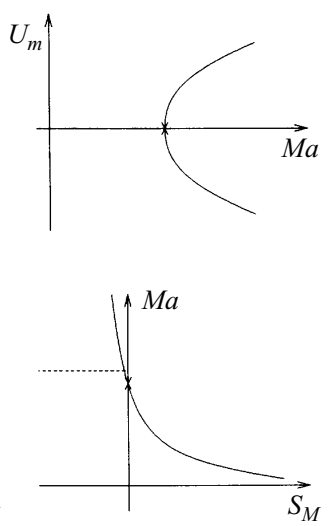

(c)
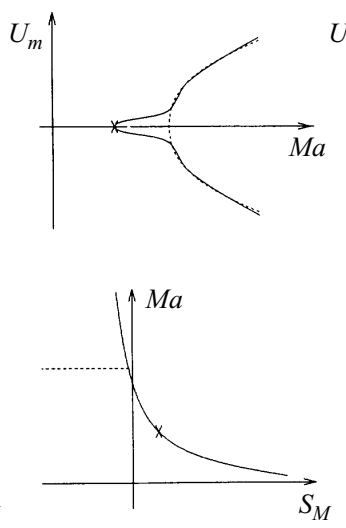

$(d)$
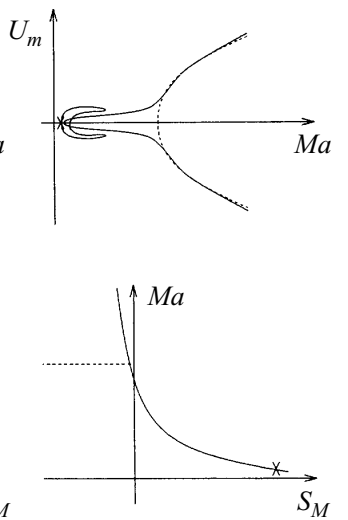

FIGURE 25. Upper figures: evolution of bifurcation diagrams and $M a_{c}$ with $S_{M}$ for $M a>0$. (a) $S_{M}<0$. Steady bifurcation branches leftward. Soret regime (weakly convecting unstable branches) and Marangoni regime (strongly convecting stable branches) separated by saddle-node bifurcation near the pure thermal threshold. (b) $S_{M}=0$. Pure thermal convection. (c) Small $S_{M}>0$. Pure thermal threshold divides Soret regime (weak convection) from Marangoni regime (strong convection). (d) Larger $S_{M}>0$. Initially bifurcating branches terminate for $M a$ below pure thermal threshold; transition to Marangoni regime occurs for subsequent branch. Dashed curves on $(a)$, $(c)$, and $(d)$ reproduce pure thermal branch from figure $(b)$. Crosses indicate primary bifurcation points, including Hopf bifurcation in $(a)$. Lower figures: thresholds $M a_{c}$ as a function of $S_{M}$. Steady bifurcation threshold shown as solid curve, Hopf bifurcation threshold as dashed curve. Crosses on each graph match bifurcations on corresponding diagrams above.

regimes. For $M a$ between the thermal-solutal and the thermal thresholds, a weak convective flow exists: this is the Soret regime. For $M a$ higher than the pure thermal threshold, the much stronger flow resembles convection in a pure fluid and the concentration field is mixed: this is the Marangoni regime. For larger $S_{M}$ (figures $25 d$ and 17), the branches which are the first to bifurcate may terminate while still in the Soret regime. In this case, the transition to the Marangoni regime is seen on other branches.

The case $S_{M}<0, M a>0$, where a stabilizing solutal influence competes with a destabilizing thermal influence, is of particular interest (figures 25a and 20). The first instability is a Hopf bifurcation, leading to oscillations. For $A=2$, these oscillations exist only over a very small range of $M a$ before being destroyed by global bifurcations. Except over this very small interval, the usual sequence is observed: one-roll steady states, succeeded by the upflowing two-roll steady state. For larger $A$, we expect features of this scenario - the interval over which there are oscillations, the number of rolls - to change quantitatively. We can again distinguish Soret and Marangoni regimes along the steady convective branches. However for negative $S_{M}$, the distinction is more complicated than for positive $S_{M}$ : the Soret branches are unstable and coexist with stable Marangoni branches to which they are connected by saddle-node bifurcations.

Detailed study of the spectrum in the $S_{M}<0, M a>0$ quadrant reveals several interesting features. In addition to the usual classification of the eigenmodes as symmetric or antisymmetric with respect to $x$-reflection, a more physical division of the spectrum also exists: under some circumstances, eigenmodes can be classified as thermal or as solutal. Whereas modes of different symmetry are uncoupled, modes 
of different physical origin are free to mix. In particular, the Hopf bifurcations involve one thermal and one solutal eigenmode, giving a concrete and quantitative interpretation to the often-stated idea that the oscillations arise from coupled solutal and thermal effects.

In summary, we have conducted the first comprehensive study of Marangoni convection with Soret effect in finite domains. By adapting a spectral-element timeintegration code to perform steady-state solving and direct calculation of bifurcation points, we have been able to obtain convection thresholds and bifurcation diagrams over a large range of aspect ratios $A$ and Marangoni Soret coefficients $S_{M}$. Complementing these calculations with temporal integration and calculation of the most unstable eigenmodes, we have been able to locate Hopf bifurcations, compute limit cycles, and confirm stability or instability of the calculated branches. We have compared our results to those from various simplified model problems - infinite aspect ratio, pure thermal, and pure solutal problems - to obtain insight into the mechanisms responsible for convection.

This research was carried out within the framework of the Centre National d'Etudes Spatiales (CNES) microgravity program and the European Human Capital and Mobility (HCM) program on Heat and Mass Transfer in Microgravity Systems. Part of this work was conducted at the University of Texas at Austin, Princeton University, and the University of California at Irvine, where partial support was provided by NFS grant No. DMS-93-08774. The authors thank R. Sani, D. Barkley, I. Kevrekides, P. J. Aubry, M. Schatz, H. L. Swinney, and E. Titi for fruitful discussions. Computations were carried out on the Cray YMP C98 and C94 of the Institut du Développement et des Ressources en Informatique Scientifique (IDRIS) sponsored by the Centre National de Recherche Scientifique (CNRS).

\section{REFERENCES}

BARten, W., LÜCKe, M., KAMPs, M. \& Schmitz, R. 1995 Convection in binary fluid mixtures. I. Extended traveling-wave and stationary states. Phys. Rev. E 51, 5636-5661.

BÉNARD, H. 1901 Les tourbillons cellulaires dans une nappe de liquide transportant de la chaleur par convection en régime permanent. Ann. Chem. Phys. 23, 62-144.

BenHadid, H. \& Roux, B. 1992 Buoyancy- and thermocapillary-driven flows in differentially heated cavities for low-Prandtl-number fluids. J. Fluid Mech. 235, 1-36.

Bensimon, D., Kolodner, P., Surko, C. M., Williams, H. \& Croquette, V. 1990 Competing and coexisting dynamical states of travelling-wave convection in an annulus. J. Fluid Mech. 217, 441-467.

Bergeon, A., Henry, D. \& BenHadid, H. 1994a Marangoni-Bénard instability in microgravity conditions with Soret effect. Intl J. Heat Mass Transfer 37, 1545-1562.

Bergeon, A., Henry, D., BenHadid, H. \& Tuckerman, L. S. $1994 b$ Stability analysis of Marangoni convection in binary mixtures subjected to the Soret effect. ASME HTD 290, 63-69.

Bergeon, A., Henry, D. \& BenHadid, H. 1995 Analytical linear stability analysis of Marangoni instability with Soret effect. Microgravity Q. 5, 123-129.

BLOCK, M. J. 1956 Surface tension as the cause of Bénard cells and surface deformation in a liquid film. Nature 178, 650.

Brand, H. R., Hohenberg P. C. \& Steinberg, V. 1984 Codimension-2 bifurcations for convection in binary fluid mixtures. Phys. Rev. A 30, 2548-2561.

Castillo, J. L. \& Velarde, M. G. 1978 Thermal diffusion and the Marangoni instability of a two component fluid layer heated from below. Phys. Lett. 66A, 489-491.

Castillo, J. L. \& Velarde, M. G. 1982 Buoyancy-thermocapillary instability: The role of interfacial deformation in one- and two-component fluid layers heated from below or above. J. Fluid Mech. 125, 463-474. 
Chen, C. F. \& Chen, C. C. 1994 Effect of surface tension on the stability of a binary fluid layer under reduced gravity. Phys. Fluids 6, 1482-1490.

Cliffe, K.A. \& Winters, K.H. 1986 The use of symmetry in bifurcation calculations and its application to the Bénard problem. J. Comput. Phys. 67, 310-327.

Cloot, A. \& Lebon, G. 1984 A non-linear analysis of the Bénard-Marangoni problem. J. Fluid Mech. 145, 447-469.

Crawford, J. D. \& KNobloch, E. 1991 Ann. Rev. Fluid Mech. 23, 341.

Cross, M. C. 1988 Structure of non-linear travelling wave states in finite geometries. Phys. Rev. Lett. A 38, 3593-3600.

DaCosta, L. N., KNobloch, E. \& Weiss, N. O. 1981 Oscillations in double-diffusive convection. J. Fluid Mech. 109, 25-44.

Davis, S. H. 1969 Buoyancy-surface tension instability by the method of energy. J. Fluid Mech. 39, 347-359.

Davis, S. H. 1987 Thermocapillary instabilities. Ann. Rev. Fluid. Mech. 19, 403-434.

Davis, S. H. \& Homsy, G. M. 1980 Energy stability theory for free surface problem: buoyancythermo-capillary layers. J. Fluid Mech. 98, 527-553.

Dauby, P. C. \& Lebon, G. 1997 Bénard-Marangoni instability in rigid rectangular containers. J. Fluid Mech. 329, 1-25.

Dauby, P. C., Lebon, G., Colinet, P. \& Legros, J. C. 1993 Hexagonal Marangoni convection in a rectangular box with slippery walls. Q. J. Mech. Appl. Maths 46, 683-707.

De Groot, S. R. \& Mazur, P. 1969 Non Equilibrium Thermodynamics. North Holland.

Dijkstra, H. A. 1992 On the structure of cellular solutions in Rayleigh-Bénard-Marangoni flows in small aspect ratio containers. J. Fluid Mech. 243, 73-102.

Dijkstra, H. A. 1995 Surface tension driven cellular patterns in three-dimensional boxes - Linear stability. Microgravity Sci. Technol. VII(4), 307-312.

Goussis, D. A. \& Kelly, R. E. 1990 On the thermocapillary instabilities in a liquid layer heated from below. Intl J. Heat Mass Transfer 33, 2237-2245.

Heinrichs, R., Ahlers, G. \& CANnell, D. S. 1987 Traveling waves and spatial variation in the convection of a binary mixture. Phys. Rev. A 35, 2761-2764.

Henry, D. \& Roux, B. 1988 Soret separation in a quasi-vertical cylinder. J. Fluid. Mech. 195, $175-200$.

Hirschberg, P. \& Knobloch, E. 1996 Mode interaction in large aspect ratio convection. Preprint.

Ho, K.-L. \& Chang, H.-C. 1988 On non-linear doubly diffusive Marangoni instability. AIChE J. 34, 705-722.

Hurle, D. T. \& Jakeman, E. 1971 Soret driven thermosolutal convection. J. Fluid Mech. 47, 667-687.

Joo, S. W. 1995 Marangoni instabilities in liquid mixtures with Soret effects. J. Fluid Mech. 293, $127-145$.

Karniadakis, G. Em., Israeli, M. \& Orszag, S. A. 1991 High-order splitting method for the incompressible Navier-Stokes equations. J. Comput, Phys. 97, 414-443.

KNobloch, E. 1980 Convection in binary mixtures. Phys. Fluids 23, 1918-1920.

KNobloch, E. 1986 Oscillatory convection in binary mixtures. Phys. Rev. A 34, 1538-1549.

Knobloch, E. \& Moore, D. R. 1988 Linear stability analysis of experimental Soret convection. Phys. Rev. A 37, 860-870.

Koschmieder, E. L. 1967 On convection under an air surface. J. Fluid Mech. 30, 9-15.

Koschmieder, E. L. \& Prahl, S. A. 1990 Surface-tension-driven Bénard convection in small containers. J. Fluid Mech. 215, 571-583.

Koschmieder, E. L. \& Switzer, D. W. 1992 The wavenumber of supercritical surface-tension-driven Bénard convection J. Fluid Mech. 240, 533-548.

Kraska, J. R. \& Sani, R. L. 1979 Finite amplitude Bénard-Rayleigh convection. Intl J. Heat Mass Transfer 22, 535-546.

Legros, J. C., Platten, J. K. \& Poty, P. G. 1972 Stability of a two component fluid layer heated from below. Phys. Fluids 15, 1383-1390.

Lhost, O. \& Platten, J. K. 1989 Large-scale convection induced by the Soret effect. Phys. Rev. A 40, 6415-6420.

MCTAGgaRT, C. L. 1983 Convection driven by concentration- and temperature-dependent surface tension. J. Fluid Mech. 134, 301-310. 
Mamun, C. K. \& Tuckerman, L. S. 1995 Asymmetry and Hopf bifurcation in spherical Couette flow. Phys. Fluids 7, 80-91.

Moses, E., Fineberg, J. \& Steinberg, V. 1987 Multistability and confined travelling wave patterns in a convecting binary mixture. Phys. Rev. A 35, 2761-2764.

NiELD, D. A. 1964 Surface tension and buoyancy effects in cellular convection. J. Fluid Mech. 19, 341-352.

Nield, D. A. 1967 The thermohaline Rayleigh-Jeffreys problem. J. Fluid Mech. 29, 545-558.

OpPe, T. C., Joubert, W. D. \& Kincaid, D. R. 1989 NSPCG, a package for solving large sparse linear systems by various iterative methods. Center for Numerical Analysis, University of Texas at Austin.

Oron, A. \& Rosenau, P. 1989 Evolution of the coupled Bénard-Marangoni convection. Phys. Rev. A 39, 2063-2069.

Patera, A. T. 1984 A spectral element method for fluid dynamics: Laminar flow in a channel expansion. J. Comput. Phys. 54, 468-488.

Pearson, J. R. A. 1958 On convection cells induced by surface tension. J. Fluid Mech. 4, 489-500.

Platten, J. K. \& Chavepeyer, G. 1973 Oscillatory motion in Bénard cell due to the Soret effect. J. Fluid Mech. 60, 305-320.

Platten, J. K. \& Chavepeyer, G. 1975 An hysteresis loop in the two component Bénard problem. Intl J. Heat Mass Transfer 18, 1071-1075.

RAYLEIGH, LORD 1916 On convective currents in a horizontal layer of fluid when the higher temperature is on the under side. Phil. Mag. 32(6), 529-546.

RehberG, I. \& Ahlers, G. 1985 Experimental observation of a codimension-2 bifurcation in a binary fluid mixture. Phys. Rev. Lett. 55, 500-503.

Riley, D. S. \& Winters, K. H. 1989 Modal exchange in Lapwood convection J. Fluid Mech. 204, 489-500.

Rosenblat, S., Davis, S. H. \& Homsy, G. M. 1982 Non-linear Marangoni convection in bounded layers. Part 2. J. Fluid Mech. 120, 91-138.

Schechter, R. S., Prigogine, I. \& Hamm, J. R. 1972 Thermal diffusion and convective instability. 1972 Phys. Fluids 15, 379-386.

SCRIVEN, L. E. \& Sternling, C. V. 1964 On cellular convection driven by surface-tension gradients: effects of mean surface tension and surface viscosity. J. Fluid Mech. 19, 321-340.

Sмiтh, K. A. 1966 On convective instability induced by surface tension gradients. J. Fluid Mech. 24, 401-414.

Thess, A. \& Orszag, S. A. 1995 Surface-tension-driven Bénard convection at infinite Prandtl number. J. Fluid Mech. 283, 201-230.

TuCKerman, L. S. 1989 Steady-state solving via Stokes preconditioning; Recursion relations for elliptic operators. In Proc. 11th Intl Conf. on Numerical Methods in Fluid Dynamics (ed. D. L. Dwoyer, M. Y. Hussaini \& R. G. Voigt). Springer.

VAN DE Vooren, A. I. \& Dijkstra, H. A. 1989 A finite element stability analysis for the Marangoni problem in a rectangular container with rigid sidewalls. Computers and Fluids 17, 467-485.

Van Vaerenbergh, S., Colinet, P., Georis, Ph. \& Legros, J. C. 1991 Preparatory results for a Marangoni-Bénard experiment with Soret effect in micro-gravity conditions. unpublished.

Veronis, G. 1968 Effect of stabilizing gradient of solute on thermal convection. J. Fluid Mech. 34, 315-336.

Walden, R. W., Kolodner, P. \& Passner, A. 1985 Travelling waves and chaos in convection in binary mixtures. Phys. Rev. Lett. 55, 496-499.

Winters, K. H., Plesser, T. H. \& Cliffe, K. A. 1988 The onset of convection in a finite container due to surface tension and buoyancy. Physica 29D, 387-401.

Zimmermann, G., Müller, U. \& Davis, S. H. 1992 Bénard convection in binary mixtures with Soret effect and solidification. J. Fluid Mech. 238, 657 -682. 